TRANSACTIONS OF THE

AMERICAN MATHEMATICAL SOCIETY

Volume 362, Number 8, August 2010, Pages 4331-4356

S 0002-9947(10)05060-9

Article electronically published on March 5, 2010

\title{
THE BOCHNER-SCHOENBERG-EBERLEIN PROPERTY FOR COMMUTATIVE BANACH ALGEBRAS, ESPECIALLY FOURIER AND FOURIER-STIELTJES ALGEBRAS
}

\author{
EBERHARD KANIUTH AND ALI ÜLGER
}

\begin{abstract}
The classical Bochner-Schoenberg-Eberlein theorem characterizes the continuous functions on the dual group of a locally compact abelian group $G$ which arise as Fourier-Stieltjes transforms of elements of the measure algebra $M(G)$ of $G$. This has led to the study of the algebra of BSE-functions on the spectrum of an arbitrary commutative Banach algebra and of the concept of a BSE-algebra as introduced by Takahasi and Hatori. Since then BSEalgebras have been studied by several authors. In this paper we investigate BSE-algebras in the general context on the one hand and, on the other hand, we specialize to Fourier and Fourier-Stieltjes algebras of locally compact groups.
\end{abstract}

\section{INTRODUCTION}

Let $A$ be a commutative Banach algebra with Gelfand spectrum $\Delta(A)$ and let $M(A)$ denote the multiplier algebra of $A$. A bounded continuous function $\sigma$ on $\Delta(A)$ is called a BSE-function if there exists a constant $C>0$ such that for any finitely many elements $\varphi_{1}, \ldots, \varphi_{n}$ of $\Delta(A)$ and complex numbers $c_{1}, \ldots, c_{n}$ the inequality

$$
\left|\sum_{j=1}^{n} c_{j} \sigma\left(\varphi_{j}\right)\right| \leq C \cdot\left\|\sum_{j=1}^{n} c_{j} \varphi_{j}\right\|_{A^{*}}
$$

holds. The BSE-norm of $\sigma,\|\sigma\|_{B S E}$, is defined to be the infimum of all such $C$. The algebra $A$ is called a $B S E$-algebra (or said to have the BSE-property) if the BSEfunctions on $\Delta(A)$ are precisely the Gelfand transforms of the elements of $M(A)$. Thus BSE-algebras admit a remarkable description of the range of the Gelfand homomorphism of $M(A)$, restricted to the subset $\Delta(A)$ of $\Delta(M(A))$.

The contraction BSE stands for Bochner-Schoenberg-Eberlein and refers to the famous theorem, proved by Bochner and Schoenberg (1934) for the additive group of real numbers and by Eberlein (1955) for general locally compact abelian groups $G$, saying that, in the above terminology, the group algebra $L^{1}(G)$ is a BSE-algebra

Received by the editors September 14, 2008 and, in revised form, January 12, 2009.

2010 Mathematics Subject Classification. Primary 46J05, 43A30; Secondary 46J10, 22E15.

Key words and phrases. Commutative Banach algebra, multiplier algebra, BSE-function, BSEalgebra, second duals, uniform algebra, unitization, locally compact group, Fourier and FourierStieltjes algebras, Lipschitz algebra.

The first author was supported by the German Research Foundation.

The second author was supported by the Turkish Academy of Sciences.

(C)2010 American Mathematical Society Reverts to public domain 28 years from publication 
(see 31]). Recall that $\Delta\left(L^{1}(G)\right)$ is naturally homeomorphic to the dual group $\widehat{G}$ of $G$ and $M\left(L^{1}(G)\right)$ is isometrically isomorphic to the measure algebra $M(G)$ of $G$.

The notion of BSE-algebra and the algebra of BSE-functions were introduced and first studied by Takahasi and Hatori [34. This paper initiated a series of subsequent publications [18, 19, 20, 35, 36] and has had a great impact. In [34, apart from a number of examples, useful characterizations of BSE-functions were given. Moreover, $C_{B S E}(\Delta(A))$, equipped with the BSE-norm, was shown to be a semisimple Banach algebra, and the relevance of the existence of a $\Delta$-weak bounded approximate identity was demonstrated. The BSE-property also appeared in 22$]$ and [37.

In this paper we investigate BSE-algebras from the general point of view on the one hand and the BSE-property for Fourier and Fourier-Stieltjes algebras of locally compact groups on the other hand. A brief outline is as follows.

In the preliminary Section 1 we present several facts about the algebra of BSEfunctions. In particular, we relate $C_{B S E}(\Delta(A))$ to $C_{B S E}(\Delta(I))$ and $C_{B S E}\left(\Delta\left(A_{e}\right)\right)$ for ideals $I$ of $A$ and the unitization $A_{e}$ of $A$. The results are used substantially later in the paper.

Suppose that $A$ has a bounded approximate identity. To every element $m$ of $A^{* *}$ we associate the function $\widehat{m}: \Delta(A) \rightarrow \mathbb{C}$ defined by $\widehat{m}(\varphi)=\langle m, \varphi\rangle$. This function $\widehat{m}$ need not be continuous on $\Delta(A)$. It turns out that the subspace

$$
C\left(A^{* *}\right)=\left\{m \in A^{* *}: \widehat{m}: \Delta \rightarrow \mathbb{C} \text { is continuous }\right\}
$$

of $A^{* *}$ plays an important role in our investigation. In Section 2, we establish two criteria for a semisimple commutative Banach algebra $A$ to be a BSE-algebra. The first one (Theorem 2.1) characterizes BSE-algebras in terms of $C\left(A^{* *}\right)$ and turns out to be useful in some of the proofs of subsequent results. The second characterization, which proves to be even more important, states that if $\|a\|=\|\widehat{a}\|_{B S E}$ holds for all $a \in A$ (which is the case for most Banach algebras), then $A$ is a BSE-algebra if and only if the Fourier-Stieltjes transform $M(A) \rightarrow C^{b}(\Delta(A))$ is a semi-embedding of Banach spaces (Theorem 2.4). Theorem 2.4 admits a surprising application to uniform algebras.

In Section 3 we study algebras $A$ which are ideals in their second duals. We obtain that then $A$ is a BSE-algebra if and only if $A$ has a bounded approximate identity and we determine the best possible norm bound (Theorem 3.1).

It is a natural question whether $M(A)$ and $A_{e}$ have to be BSE-algebras whenever $A$ is a BSE-algebra, and vice versa. Regarding the unitization, we give in Theorem 4.8 a necessary and sufficient condition in terms of $A$ for $A_{e}$ to be a BSE-algebra. We also comment on when this condition is satisfied. Suppose again that $A$ has a bounded approximate identity. Then, if $M(A)$ is a BSE-algebra, so is $A$ at least when $A \rightarrow C_{B S E}(\Delta(A))$ is isometric (Theorem 4.1). The reverse implication holds if $\Delta(A)$ is dense in $\Delta(M(A))$ (Corollary 4.3).

Several of these results are applied in Section 5, where we investigate the Fourier and Fourier-Stieltjes algebras, $A(G)$ and $B(G)$, associated with an arbitrary locally compact group $G$. We remind the reader that when $G$ is abelian, $A(G)$ and $B(G)$ are isometrically isomorphic to $L^{1}(\widehat{G})$ and $M(\widehat{G})$, respectively. The Fourier algebra $A(G)$ turns out to be a BSE-algebra if and only if the group $G$ is amenable, and in this case the closed ideals of $A(G)$ which are BSE-algebras are found to be precisely those ideals which possess bounded approximate identities and therefore correspond to the closed sets in the coset ring of $G$ (Theorems 5.1 and 5.3). Moreover, if $I$ 
is such an ideal, then the elements of $I$ can be identified within the algebra of BSE-functions on $\Delta(I)$ (Theorem 5.4). If $G$ is abelian, then $B(G)$ is a BSE-algebra (if and) only if $G$ is compact, and this result extends to nilpotent groups. For general $G$, however, there is a somewhat surprising diversity in that, no matter whether $G$ is amenable or not, $B(G)$ can be a BSE-algebra and can fail to be one (see Theorems 5.7 and 5.9). All these features are illustrated by examples. Most notably, the Fourier-Stieltjes algebras of noncompact connected simple Lie groups with finite centre and of certain 2-step solvable groups are BSE-algebras.

\section{Preliminaries and the Algebra of BSE-Functions}

Let $A$ be a commutative Banach algebra. A linear operator $T$ on $A$ is called a multiplier if it satisfies $x T(y)=T(x y)$ for all $x, y \in A$. The set $M(A)$ of all multipliers of $A$ is a unital commutative Banach algebra, the multiplier algebra of $A$. For each $T \in M(A)$ there exists a unique continuous function $\widehat{T}$ on $\Delta(A)$ such that $\widehat{T(a)}(\varphi)=\widehat{T}(\varphi) \widehat{a}(\varphi)$ for all $a \in A$ and $\varphi \in \Delta(A)$ [24, Theorem 1.2.2].

A bounded net $\left(e_{\alpha}\right)_{\alpha}$ in $A$ is called a $\Delta$-weak bounded approximate identity if it satisfies $\varphi\left(e_{\alpha}\right) \rightarrow 1$ (equivalently, $\varphi\left(e_{\alpha} a\right) \rightarrow \varphi(a)$ for every $a \in A$ ) for all $\varphi \in \Delta(A)$. Such approximate identities were studied in [21, where the first example was given of a semisimple commutative Banach algebra which has a $\Delta$-weak approximate identity, but does not possess a bounded approximate identity. Accordingly, in 34 these approximate identities are referred to as bounded approximate identities in the sense of Jones and Lahr, but we prefer to stick to the above more suggestive name used in [12]. As shown in [34, Corollary 5], $A$ has a $\Delta$-weak bounded approximate identity if and only if $\widehat{M(A)} \subseteq C_{B S E}(\Delta(A))$. There are classes of semisimple commutative Banach algebras for which the existence of a $\Delta$-weak bounded approximate identity already forces $\widehat{M(A)}=C_{B S E}(\Delta(A))$, that is, $A$ is a BSE-algebra, but which nevertheless don't admit bounded approximate identities [18. We shall mention these at the end of this section. In many cases, however, the three properties that $A$ is a BSE-algebra, $A$ has a bounded approximate identity, and $A$ has a $\Delta$-weak bounded approximate identity turn out to be equivalent (see Theorems 3.1, 5.1 and 5.3).

The multiplier algebra of a semisimple BSE-algebra $A$ is topologically isomorphic to $C_{B S E}(\Delta(A))$ [34, Corollary 6]; that is, there exist positive real numbers $c$ and $d$ such that $c\|T\| \leq\|\widehat{T}\|_{B S E} \leq d\|T\|$ for all $T \in M(A)$. In particular, the norms $a \rightarrow\|a\|$ and $a \rightarrow\|\widehat{a}\|_{B S E}$ on $A$ are equivalent. Since we always have $\|\widehat{a}\|_{B S E} \leq\|a\|$, it is an interesting question of when these two norms on $A$ are equal. A number of examples where this happens to be the case are given in [36], among them $L^{1}$ algebras of locally compact abelian groups, $L^{p}(G)$ for a compact abelian group $(1 \leq p<\infty)$ and commutative $H^{*}$-algebras.

For a commutative Banach algebra $A$ we define $\beta(A) \in[0, \infty]$ by

$$
\beta(A)=\sup \left\{\left|\sum_{j=1}^{n} c_{j}\right|:\left\|\sum_{j=1}^{n} c_{j} \varphi_{j}\right\|_{A^{*}} \leq 1, \varphi_{j} \in \Delta(A), c_{j} \in \mathbb{C}\right\} .
$$

It is clear from the very definition that $\beta(A)$ is finite if and only if the algebra $C_{B S E}(\Delta(A))$ is unital; that is, the constant function 1 on $\Delta(A)$ is a BSE-function. Moreover, in this case, $\beta(A)=\|1\|_{B S E}$. 
Lemma 1.1. Let $A$ be a commutative Banach algebra. Then the algebra $C_{B S E}(\Delta(A))$ is unital if and only if $A$ has a $\Delta$-weak bounded approximate identity. Furthermore, in this case, the norm $\|1\|_{B S E}=\beta(A)$ is equal to the minimum of the norm bounds of all such approximate identities.

Proof. Suppose that $\left(e_{\alpha}\right)_{\alpha}$ is a $\Delta$-weak bounded approximate identity. Then, for any $\sum_{j=1}^{n} c_{j} \varphi_{j} \in \operatorname{span}(\Delta(A))$,

$$
\begin{aligned}
\left|\sum_{j=1}^{n} c_{j}\right| & =\lim _{\alpha}\left|\sum_{j=1}^{n} c_{j} \varphi_{j}\left(e_{\alpha}\right)\right|=\lim _{\alpha}\left|\left\langle e_{\alpha}, \sum_{j=1}^{n} c_{j} \varphi_{j}\right\rangle\right| \\
& \leq \sup _{\alpha}\left\|e_{\alpha}\right\| \cdot\left\|\sum_{j=1}^{n} c_{j} \varphi_{j}\right\|_{A^{*}} .
\end{aligned}
$$

So $1 \in C_{B S E}(\Delta(A))$ and $\beta(A) \leq \sup _{\alpha}\left\|e_{\alpha}\right\|$.

Conversely, let $1 \in C_{B S E}(\Delta(A))$. Then, for all $\varphi_{1}, \ldots, \varphi_{n} \in \Delta(A)$ and $c_{1}, \ldots, c_{n}$ $\in \mathbb{C}$

$$
\left|\sum_{j=1}^{n} c_{j}\right| \leq \beta(A) \cdot\left\|\sum_{j=1}^{n} c_{j} \varphi_{j}\right\|_{A^{*}} .
$$

This means that the assignment $\sum_{j=1}^{n} c_{j} \varphi_{j} \rightarrow \sum_{j=1}^{n} c_{j}$ is a bounded linear functional on $\operatorname{span}(\Delta(A))$. By the Hahn-Banach theorem there exists $m \in A^{* *}$ such that $\|m\|_{A^{* *}} \leq \beta(A)$ and $\langle m, \varphi\rangle=1$ for all $\varphi \in \Delta(A)$. Since the unit ball of $A$ is $w^{*}$-dense in the unit ball of $A^{* *}$, there exists a net $\left(e_{\alpha}\right)_{\alpha}$ in $A$ with $e_{\alpha} \rightarrow m$ in the $w^{*}$-topology and $\left\|e_{\alpha}\right\| \leq\|m\|$ for all $\alpha$. In particular, $\varphi\left(e_{\alpha}\right) \rightarrow\langle m, \varphi\rangle=1$ for all $\varphi \in \Delta(A)$. So $\left(e_{\alpha}\right)_{\alpha}$ is a $\Delta$-weak bounded approximate identity and, combining this with the first part of the proof, gives

$$
\beta(A) \leq \sup _{\alpha}\left\|e_{\alpha}\right\| \leq\|m\| \leq \beta(A) .
$$

This finishes the proof of the lemma.

Lemma 1.2. Let $A$ and $B$ be commutative Banach algebras and $\phi$ a continuous homomorphism from $A$ onto $B$ and let $\widehat{\phi}=\left.\phi^{*}\right|_{\Delta(B)}: \Delta(B) \rightarrow \Delta(A)$. If $\sigma \in$ $C_{B S E}(\Delta(A))$, then $\sigma \circ \widehat{\phi} \in C_{B S E}(\Delta(B))$ and

$$
\|\sigma \circ \widehat{\phi}\|_{B S E} \leq\|\sigma\|_{B S E}\|\phi\| \text {. }
$$

Proof. For $\varphi_{1}, \ldots, \varphi_{n} \in \Delta(A)$ and $c_{1}, \ldots, c_{n} \in \mathbb{C}$, we have

$$
\begin{aligned}
\left.\mid \sum_{j=1}^{n} c_{j}(\sigma \circ \widehat{\phi}) \varphi_{j}\right) \mid & =\left|\sum_{j=1}^{n} c_{j} \sigma\left(\varphi_{j} \circ \phi\right)\right| \\
& \leq\|\sigma\|_{B S E} \cdot\left\|\sum_{j=1}^{n} c_{j}\left(\varphi_{j} \circ \phi\right)\right\|_{A^{*}} \\
& \leq\|\sigma\|_{B S E} \cdot\left\|\sum_{j=1}^{n} c_{j} \varphi_{j}\right\|_{B^{*}} \cdot\|\phi\|,
\end{aligned}
$$

as claimed. 
Corollary 1.3. Let $A$ and $B$ be unital commutative Banach algebras. If $A$ and $B$ are topologically isomorphic, then $A$ is a BSE-algebra if and only if $B$ is a BSEalgebra.

Proof. Let $\phi: A \rightarrow B$ be a topological isomorphism and suppose that $B$ is a BSE-algebra. With the notation of Lemma 1.2, if $\sigma \in C_{B S E}(\Delta(A))$, then $\sigma \circ \widehat{\phi} \in$ $C_{B S E}(\Delta(B))$ and hence there exists $b \in B$ such that, for any $\varphi \in \Delta(B)$,

$$
\sigma(\varphi \circ \phi)=(\sigma \circ \widehat{\phi})(\varphi)=\widehat{b}(\varphi)=(\varphi \circ \phi)\left(\phi^{-1}(b)\right)
$$

Since $\Delta(A)=\{\varphi \circ \phi: \varphi \in \Delta(B)\}$, it follows that $\widehat{\phi^{-1}(b)}=\sigma$. Thus $C_{B S E}(\Delta(A)) \subseteq$ $\widehat{A}$ and so $A$ is a BSE-algebra.

Lemma 1.4. Let $A$ be a commutative Banach algebra and $\sigma \in C_{B S E}(\Delta(A))$. Let $I$ be a closed ideal of $A$, and suppose that $I$ has a $\Delta$-weak approximate identity, bounded by $c>0$. Then $\left.\sigma\right|_{\Delta(I)} \in C_{B S E}(\Delta(I))$ and

$$
\left\|\left.\sigma\right|_{\Delta(I)}\right\|_{B S E} \leq c\|\sigma\|_{B S E}
$$

Proof. We have to show that given $\epsilon>0$, for any $\varphi_{1}, \ldots, \varphi_{n} \in \Delta(I)$ and $c_{1}, \ldots, c_{n}$ $\in \mathbb{C}$

$$
\left|\sum_{j=1}^{n} c_{j} \sigma\left(\varphi_{j}\right)\right| \leq c\|\sigma\|_{B S E} \cdot\left\|\sum_{j=1}^{n} c_{j} \varphi_{j}\right\|_{I^{*}}+\epsilon .
$$

First, select $a \in A$ such that $\|a\| \leq 1$ and

$$
\left\|\sum_{j=1}^{n} c_{j} \varphi_{j}\right\|_{A^{*}} \leq\left|\sum_{j=1}^{n} c_{j} \varphi_{j}(a)\right|+\frac{\epsilon}{2\|\sigma\|_{B S E}} .
$$

Next choose $0<\delta<1$ such that

$$
\frac{\delta}{1-\delta} \sum_{j=1}^{n}\left|c_{j}\right| \leq \frac{\epsilon}{2 c\|\sigma\|_{B S E}}
$$


By hypothesis, there exists $b \in I$ such that $\|b\| \leq c$ and $\left|\varphi_{j}(b)-1\right| \leq \delta$ for $j=1, \ldots, n$. Then we get

$$
\begin{aligned}
\left|\sum_{j=1}^{n} c_{j} \sigma\left(\varphi_{j}\right)\right| & \leq\|\| \sigma\left\|_{B S E} \cdot \sum_{j=1}^{n} c_{j} \varphi_{j}\right\|_{A^{*}} \\
& \leq\|\sigma\|_{B S E}\left(\left|\sum_{j=1}^{n} c_{j} \varphi_{j}(a)\right|+\frac{\epsilon}{2\|\sigma\|_{B S E}}\right) \\
& =\|\sigma\|_{B S E}\left(\left|\sum_{j=1}^{n} \frac{c_{j}}{\varphi_{j}(b)} \varphi_{j}(b a)\right|+\frac{\epsilon}{2\|\sigma\|_{B S E}}\right) \\
& \leq\|\sigma\|_{B S E}\left(\left|\sum_{j=1}^{n} c_{j} \varphi_{j}(b a)\right|+\sum_{j=1}^{n}\left|\frac{c_{j}\left(\varphi_{j}(b)-1\right)}{\varphi_{j}(b)} \varphi_{j}(b a)\right|+\frac{\epsilon}{2\|\sigma\|_{B S E}}\right) \\
& \leq\|\sigma\|_{B S E}(\|b a\| \\
& \left.\left.\leq c\|\sigma\|_{B S E} \cdot\left\|\sum_{j=1}^{n} c_{j} c_{j} \varphi_{j}\right\|_{I^{*}}+\sum_{j=1}^{n} \frac{\left|c_{j}\right| \delta}{1-\delta}\right)+\frac{\epsilon}{2\|\sigma\|_{B S E}}\right)
\end{aligned}
$$

and we are done.

Lemma 1.5. Let $A$ be a commutative Banach algebra and let $I$ be a closed ideal of $A$ such that $A / I$ is semisimple. For a complex-valued function $\sigma$ on $\Delta(I)$, let $\widetilde{\sigma}: \Delta(A) \rightarrow \mathbb{C}$ be defined by $\widetilde{\sigma}(\varphi)=\sigma(\varphi)$ for $\varphi \in \Delta(A)$ and $\widetilde{\sigma}(\varphi)=0$ for $\varphi \notin \Delta(I)$.

(i) If $\sigma \in C_{B S E}(\Delta(I)) \cap C_{0}(\Delta(I))$, then $\widetilde{\sigma} \in C_{B S E}(\Delta(A))$ and $\|\widetilde{\sigma}\|_{B S E}=$ $\|\sigma\|_{B S E}$.

(ii) If $C_{B S E}(\Delta(A)) \subseteq \widehat{M(A)}$, then $C_{B S E}(\Delta(I)) \cap C_{0}(\Delta(I)) \subseteq \widehat{M(I)}$.

If $\Delta(I)$ is closed in $\Delta(A)$, the same conclusions hold if $C_{B S E}(\Delta(I)) \cap$ $C_{0}(\Delta(I))$ is replaced by $C_{B S E}(\Delta(I))$.

Proof. The proof of both, (i) and (ii), is only a slight modification of the arguments in the proof of [34, Theorem 8].

Let $G$ be an abelian locally compact group and $S$ a Segal algebra on $G$. In [18, Lemma 1.1] it was shown that $S$ is a BSE-algebra if (and only if) $S$ has a $\Delta$ weak bounded approximate identity. But note that $S$ has a bounded approximate identity only if $S=L^{1}(G)$. Now, in [18, two classes of Segal algebras were shown to admit $\Delta$-weak bounded approximate identities.

(1) For $1<p \leq \infty$, let $S_{p}(G)=L^{1}(G) \cap L^{p}(G)$ with norm defined to be $\|f\|=\max \left\{\|f\|_{1},\|f\|_{p}\right\}$. Then $S_{p}(G)$ has a $\Delta$-weak approximate identity of norm 1 whenever $G$ is non-compact [18, Theorem 2.1].

(2) For $1<p<\infty$, let $A_{p}(G)=\left\{f \in L^{1}(G): \widehat{f} \in L^{p}(\widehat{G})\right\}$ with norm $\|f\|=$ $\max \left\{\|f\|_{1},\|\widehat{f}\|_{p}\right\}$. If $G$ is non-compact and non-discrete, then $A_{p}(G)$ has a $\Delta$-weak approximate identity of norm 1 [18, Theorem 3.1]. 


\section{Characterizations of BSE-Algebras}

In this section we present two characterizations of semisimple BSE-algebras with bounded approximate identities both of which turn out to be quite useful. Moreover, we feel that they clearly display those properties of $A$ and of its multiplier algebra $M(A)$ that are relevant in our context. In the entire paper, the second dual $A^{* *}$ of a commutative Banach algebra $A$ will always be equipped with the first Arens product, which is defined as follows (compare [2, Section 9]). For $a, b \in A, f \in A^{*}$ and $m, n \in A^{* *}$, the elements $f \cdot a$ and $m \cdot f$ of $A^{*}$ and $m n \in A^{* *}$ are defined by

$$
\langle f \cdot a, b\rangle=\langle f, a b\rangle,\langle m \cdot f, b\rangle=\langle m, f \cdot b\rangle \text { and }\langle m n, f\rangle=\langle m, n \cdot f\rangle,
$$

respectively. With this multiplication, $A^{* *}$ is a Banach algebra and $A$ is a subalgebra of $A^{* *}$. In general, the multiplication $(m, n) \rightarrow m n$ is not separately continuous with respect to the $w^{*}$-topology on $A^{* *}$. But, for fixed $n \in A^{* *}$, the mapping $m \rightarrow m n$ is $w^{*}$-continuous. Although under this multiplication $A^{* *}$ is not commutative in general, commutativity of $A$ implies that $a m=m a$ for all $m \in A^{* *}$ and $a \in A$.

If $\sigma \in C_{B S E}(\Delta(A))$, then the linearization $\widetilde{\sigma}: \operatorname{span}(\Delta(A)) \rightarrow \mathbb{C}$ of $\sigma$, defined by $\widetilde{\sigma}\left(\sum_{j=1}^{n} c_{j} \varphi_{j}\right)=\sum_{j=1}^{n} c_{j} \sigma\left(\varphi_{j}\right)$ for $c_{1}, \ldots, c_{n} \in \mathbb{C}$ and $\varphi_{1}, \ldots, \varphi_{n} \in \Delta(A)$, is a bounded linear functional and hence by the Hahn-Banach theorem there exists $m \in A^{* *}$ such that $\|m\|=\|\sigma\|_{B S E}$ and $\widehat{m}(\varphi)=\langle m, \varphi\rangle=\sigma(\varphi)$ for all $\varphi \in \Delta(A)$. The essential point here is that $\widehat{m}$ is continuous on $\Delta(A)$. This leads us to introduce the set

$$
C\left(A^{* *}\right)=\left\{m \in A^{* *}: \widehat{m}: \Delta \rightarrow \mathbb{C} \text { is continuous }\right\} .
$$

Then $C\left(A^{* *}\right)$ is a norm closed subalgebra of $A^{* *}$. By Theorem 4 of [34], $\widehat{C\left(A^{* *}\right)}=$ $C_{B S E}(\Delta(A))$. If $m_{1}$ and $m_{2}$ are two extensions of $\widetilde{\sigma}$, then $\widehat{m_{1}-m_{2}}=0$ on $\Delta(A)$. Therefore, a naturally arising space is

$$
R\left(A^{* *}\right)=\left\{m \in A^{* *}: \widehat{m}=0 \text { on } \Delta(A)\right\} .
$$

The space $R\left(A^{* *}\right)$ is a $w^{*}$-closed ideal of $A^{* *}$.

Suppose that $A$ has a bounded approximate identity and hence $A^{* *}$ has a right identity $e$. Then the map $j$ from $M(A)$ into $A^{* *}$ defined by $j(T)=T^{* *}(e)$ is a homomorphism with $\|j(T)\| \leq\|e\| \cdot\|T\|$. Conversely,

$$
\begin{aligned}
\|j(T)\| & =\sup \left\{\left|\left\langle T^{* *}(e), f\right\rangle\right|: f \in A_{1}^{*}\right\} \\
& \geq \sup \left\{\left|\left\langle T^{* *}(e), a \cdot f\right\rangle\right|: a \in A_{1}, f \in A_{1}^{*}\right\} \\
& =\sup \left\{|\langle T(a), f\rangle|: a \in A_{1}, f \in A_{1}^{*}\right\} \\
& =\sup \left\{\|T(a)\|: a \in A_{1}\right\}=\|T\| .
\end{aligned}
$$

So $j$ is a Banach algebra isomorphism between $M(A)$ and the closed subalgebra $j(M(A))$ of $A^{* *}$, and $j$ is an isometry if $\|e\|=1$.

Our first result characterizes BSE-algebras in terms of the space $C\left(A^{* *}\right)$.

Theorem 2.1. Let $A$ be a semisimple commutative Banach algebra with bounded approximate identity. Then $A$ is a BSE-algebra if and only if

$$
C\left(A^{* *}\right)=j(M(A)) \oplus R\left(A^{* *}\right) \text {. }
$$

Proof. Suppose first that $A$ is a BSE-algebra and let $T \in M(A)$ and put $m=$ $T^{* *}(e) \in A^{* *}$. Then $\widehat{m}=\widehat{T}$ on $\Delta(A)$, so that $\widehat{m}$ is continuous on $\Delta(A)$. Consequently, $j(M(A)) \subseteq C\left(A^{* *}\right)$. Now, let $n$ be an arbitrary element of $C\left(A^{* *}\right)$. Then, 
since $A$ is a BSE-algebra, $\widehat{n} \in C_{B S E}(\Delta(A))$ and hence $\widehat{n}=\widehat{T}$ for some $T \in M(A)$. Let $m=T^{* *}(e) \in j(M(A))$. Then $\widehat{n-m}=0$ on $\Delta(A)$ and so $n-m \in R\left(A^{* *}\right)$. This shows that $C\left(A^{* *}\right) \subseteq j(M(A))+R\left(A^{* *}\right)$. Clearly, $j(M(A)) \cap R\left(A^{* *}\right)=\{0\}$, whence $C\left(A^{* *}\right)=j(M(A)) \oplus R\left(A^{* *}\right)$.

Conversely, if $C\left(A^{* *}\right)=j(M(A))+R\left(A^{* *}\right)$ holds, then $\widehat{M(A)}=\widehat{C\left(A^{* *}\right)}=$ $C_{B S E}(\Delta(A))$. Hence $A$ is a BSE-algebra.

As an immediate consequence of this characterization we get

Corollary 2.2. Let $A$ be a unital semisimple commutative Banach algebra. Then $A$ is a BSE-algebra if and only if $C\left(A^{* *}\right)=A \oplus R\left(A^{* *}\right)$.

The following lemma is straightforward. We include it for completeness.

Lemma 2.3. Let $E$ and $F$ be Banach spaces and let $T: E \rightarrow F$ be a bounded linear operator. Let $m \in E^{* *}$ be such that $T^{* *}(m) \in F$. If $\left(a_{\alpha}\right)_{\alpha}$ is a net in $E$ converging to $m$ in the $w^{*}$-topology of $E^{* *}$, then the net $\left(T\left(a_{\alpha}\right)\right)_{\alpha}$ converges weakly to $T^{* *}(m)$ in $F$.

To motivate the next characterization of BSE-algebras, consider the measure algebra $M(G)$ of a locally compact abelian group $G$ and the Fourier-Stieltjes transform $\Gamma: M(G) \rightarrow C^{b}(\widehat{G})$. Then $\Gamma\left(M(G)_{1}\right)$ is norm closed in $C^{b}(\widehat{G})$ 31, Theorem 1.9.2]. In terms of general Banach space theory, $\Gamma$ is a semi-embedding. Recall that a bounded linear operator $T: E \rightarrow F$ between Banach spaces $E$ and $F$ is said to be a semi-embedding if $T$ is one-to-one and $T\left(E_{1}\right)$, the image of the closed unit ball of $E$, is closed in $F$. Of course, if $T$ is a topological isomorphism onto its range, then $T$ is a semi-embedding. However, being a semi-embedding is a much weaker condition. The reader can find ample information on this notion in [3].

The following theorem shows that in the context of general commutative Banach algebras $A$, the relevant condition for $A$ to be a BSE-algebra is that the FourierStieltjes transform $\Gamma: M(A) \rightarrow C^{b}(\Delta(A))$ is a semi-embedding.

Theorem 2.4. Let $A$ be a semisimple commutative Banach algebra with bounded approximate identity and let $\Gamma: M(A) \rightarrow C^{b}(\Delta(A))$ denote the Fourier-Stieltjes transform. If $\Gamma$ is a semi-embedding, then $A$ is a BSE-algebra, and the converse is true at least when $\|\widehat{a}\|_{B S E}=\|a\|$ holds for all $a \in A$.

Proof. Suppose first that $\Gamma$ is a semi-embedding. To show that $A$ is a BSE-algebra we shall use Theorem 2.1. To that end, let $m \in C\left(A^{* *}\right)$ be given. Of course, we can assume that $\|m\| \leq 1$. Then there exists a net $\left(a_{\alpha}\right)_{\alpha}$ in the unit ball $A_{1}$ of $A$ such that $a_{\alpha} \rightarrow m$ in the $w^{*}$-topology of $A^{* *}$. For each $\alpha$, consider the multiplier $T_{\alpha}=L_{a_{\alpha}} \in M(A)_{1}$. Since $\widehat{m} \in C^{b}(\Delta(A))$, an application of Lemma 2.3 to the Gelfand transform shows that $\widehat{a_{\alpha}} \rightarrow \widehat{m}$ weakly in $C^{b}(\Delta(A))$. Because the functions $\widehat{T_{\alpha}}$ and $\widehat{a_{\alpha}}$ agree on $\Delta(A)$, this means that $\widehat{T_{\alpha}} \rightarrow \widehat{m}$ weakly in $C^{b}(\Delta(A))$. Therefore, the set $\Gamma\left(M(A)_{1}\right)$ being convex and norm closed, we get that $\widehat{m} \in \Gamma\left(M(A)_{1}\right)$. Thus $\widehat{m}=\widehat{T}$ for some $T \in M(A)_{1}$. Since $A$ is semisimple, $T$ is uniquely determined. Now let $e$ be a right identity in $A^{* *}$ and let $n=T^{* *}(e)$. Then $m-n$ belongs to $R\left(A^{* *}\right)$ and therefore $m \in j(M(A))+R\left(A^{* *}\right)$. This shows that $C\left(A^{* *}\right)=j(M(A)) \oplus R\left(A^{* *}\right)$ and hence $A$ is a BSE-algebra by Theorem 2.1.

Conversely, suppose that $A$ is a BSE-algebra and that $\|\widehat{a}\|_{B S E}=\|a\|$ holds for all $a \in A$. To prove that $\Gamma\left(M(A)_{1}\right)$ is norm closed in $C^{b}(\Delta(A))$, let $\sigma \in C^{b}(\Delta(A))$ be such that $\sigma$ is in the norm closure of $\Gamma\left(M(A)_{1}\right)$ in $C^{b}(\Delta(A))$. There exists a 
sequence $\left(T_{n}\right)_{n}$ in $M(A)_{1}$ such that $\widehat{T_{n}} \rightarrow \sigma$ uniformly on $\Delta(A)$ as $n \rightarrow \infty$. Let $e$ be a right identity in $A^{* *}$ and $S \rightarrow S^{* *}(e)$ the corresponding embedding of $M(A)$ into $A^{* *}$. Then, for any $\sum_{j=1}^{n} c_{j} \varphi_{j} \in \operatorname{span}(\Delta(A))$,

$$
\begin{aligned}
\left|\sum_{j=1}^{n} c_{j} \widehat{T_{n}}\left(\varphi_{j}\right)\right| & =\left|\sum_{j=1}^{n} c_{j} \widehat{T_{n}^{* *}(e)}\left(\varphi_{j}\right)\right| \\
& \leq\left\|T_{n}^{* *}(e)\right\| \cdot\left\|\sum_{j=1}^{n} c_{j} \varphi_{j}\right\|_{A^{*}} \\
& \leq\|e\| \cdot\left\|\sum_{j=1}^{n} c_{j} \varphi_{j}\right\|_{A^{*}}
\end{aligned}
$$

since $\left\|T_{n}\right\| \leq 1$. Letting $n \rightarrow \infty$, we get

$$
\left|\sum_{j=1}^{n} c_{j} \sigma\left(\varphi_{j}\right)\right| \leq\|e\| \cdot\left\|\sum_{j=1}^{n} c_{j} \varphi_{j}\right\|_{A^{*}} .
$$

This shows that $\sigma \in C_{B S E}(\Delta(A))$, and since $A$ is a BSE-algebra, there exists $T \in M(A)$ such that $\widehat{T}=\sigma$.

It remains to show that $\|T\| \leq 1$. To this end, let $a \in A$ be given. Since $\widehat{T_{n}(a)}(\varphi) \rightarrow \widehat{T(a)}(\varphi)$ for all $\varphi \in \Delta(A)$, we have for any $\sum_{j=1}^{n} c_{j} \varphi_{j} \in \operatorname{span}(\Delta(A))$,

$$
\begin{aligned}
\left|\sum_{j=1}^{n} c_{j} \widehat{T(a)}\left(\varphi_{j}\right)\right| & =\lim _{n \rightarrow \infty}\left|\sum_{j=1}^{n} c_{j} \widehat{T_{n}(a)}\left(\varphi_{j}\right)\right| \\
& =\lim _{n \rightarrow \infty}\left|\left\langle T_{n}(a), \sum_{j=1}^{n} c_{j} \varphi_{j}\right\rangle\right| \\
& \leq \limsup _{n \rightarrow \infty}\left\|T_{n}(a)\right\| \cdot\left\|\sum_{j=1}^{n} c_{j} \varphi_{j}\right\|_{A^{*}} \\
& \leq\|a\| \cdot\left\|\sum_{j=1}^{n} c_{j} \varphi_{j}\right\|_{A^{*}},
\end{aligned}
$$

so that $\|\widehat{T(a)}\|_{B S E} \leq\|a\|$. Now, by hypothesis, $\|\widehat{x}\|_{B S E}=\|x\|$ for all $x \in A$. It follows that

$$
\|T(a)\|=\|\widehat{T(a)}\|_{B S E} \leq\|a\|,
$$

and hence $\|T\| \leq 1$ since $a \in A$ was arbitrary.

Remark 2.5. Let $A$ be a semisimple commutative Banach algebra with bounded approximate identity satisfying the condition $\|\widehat{a}\|_{B S E}=\|a\|$ for all $a \in A$. In view of Theorem 2.4 one might wonder whether $A$ is a BSE-algebra if and only if the Gelfand transform $\Gamma: A \rightarrow C_{0}(\Delta(A))$, as opposed to the Fourier-Stieltjes transform, is a semi-embedding. However, the following example shows that this 
is not true. Let $G$ be any non-discrete locally compact abelian group. Then there exists a singular measure $\mu \in M(G)$ such that $\widehat{\mu} \in C_{0}(\widehat{G})$ (see [14, Theorem 7.4.1]).

Now let $G$ be compact. It is worth mentioning that in this case a functional analytic proof for the existence of such a measure $\mu$ has been given in [3]. Of course, we can assume that $\|\mu\| \leq 1$. Let $\left(u_{\alpha}\right)_{\alpha}$ be a bounded approximate identity in $L^{1}(G)$ with $\left\|u_{\alpha}\right\|_{1} \leq 1$ for all $\alpha$, and let $f_{\alpha}=u_{\alpha} * \mu \in L^{1}(G)_{1}$. Then $\widehat{f_{\alpha}} \rightarrow \widehat{\mu}$ pointwise on the discrete group $\widehat{G}$ and since $c_{0}(\widehat{G})^{*}=l^{1}(\widehat{G})$, this implies that $\widehat{f_{\alpha}} \rightarrow \widehat{\mu}$ weakly in $c_{0}(\widehat{G})$. It follows that $\widehat{\mu}$ is contained in the norm closure of the convex set $\Gamma\left(L^{1}(G)_{1}\right)$ in $c_{0}(\widehat{G})$. This shows that $\Gamma\left(L^{1}(G)_{1}\right)$ is not closed in $C^{b}(\widehat{G})$ because otherwise we would have $\widehat{\mu}=\widehat{g}$ for some $g \in L^{1}(G)$, which is impossible since $\mu$ is singular. However, recall that $\|\widehat{f}\|_{B S E}=\|f\|_{1}$ for all $f \in L^{1}(G)$.

Employing Morera's theorem and Montel's theorem, it was shown in 34, Theorem 7] that the disc algebra and the classical Hardy algebra are BSE-algebras (in this context, compare also [20]). This raises the question of whether every uniform algebra is a BSE-algebra. Here by a uniform algebra we mean a unital commutative Banach algebra which is isometrically isomorphic to a closed subalgebra of $C(X)$ for some compact Hausdorff space $X$. As a reference to uniform algebras we mention the monograph 33. A straightforward application of Theorem 2.4 shows that the above question has an affirmative answer.

Theorem 2.6. Every uniform algebra is a BSE-algebra.

Proof. Let $A$ be a uniform algebra. By Corollary 1.3, we can assume that $A$ is a closed subalgebra of $C(X)$ for some compact Hausdorff space $X$. Then the Gelfand homomorphism $\Gamma$ is an isometric isomorphism of $A$ into $C(\Delta(A))$. Moreover, $\|a\|=$ $\|\widehat{a}\|_{B S E}$ for all $a \in A$ and $\Gamma$ is a semi-embedding. So $A$ is a BSE-algebra by Theorem 2.4 .

\section{Algebras Which ARE IDEALS IN Their SECOND DUALS}

One class of commutative Banach algebras $A$, for which a particularly simple criterion for $A$ to be a BSE-algebra can be given, is the class of semisimple commutative Banach algebras which are ideals in their second duals (Theorem 3.1). For a discussion of which algebras belong to that class, see Proposition 3.5. A criterion analogous to Theorem 3.1 will turn out to be valid for ideals in Fourier algebras of amenable locally compact groups, which we address in Section 5.

Theorem 3.1. Let $A$ be a semisimple commutative Banach algebra which is an ideal in its second dual $A^{* *}$. Then the following are equivalent.

(i) $A$ is a BSE-algebra.

(ii) A has a $\Delta$-weak bounded approximate identity.

(iii) A has a bounded approximate identity.

The approximate identity in (iii) can be chosen to be bounded in norm by $\beta(A)$.

Proof. (i) $\Rightarrow$ (ii) being obvious, let (ii) hold. Then, by Lemma 1.1, $A$ has a $\Delta$-weak bounded approximate identity, say $\left(g_{\gamma}\right)_{\gamma}$. Let $e \in A^{* *}$ be a $w^{*}$-cluster point of $\left(g_{\gamma}\right)_{\gamma}$. Then $\|e\| \leq \beta(A)$, and $\widehat{e}=1$ on $\Delta(A)$ and hence $\widehat{a e}=\widehat{a}$ on $\Delta(A)$ for all $a \in A$. Since $A$ is an ideal in $A^{* *}$ and $A$ is semisimple, this implies that $a e=a$ for all $a \in A$. By Goldstein's theorem, there exists a net $\left(f_{\beta}\right)_{\beta}$ in $A$ such that $\left\|f_{\beta}\right\| \leq\|e\|$ for all $\beta$ and $f_{\beta} \rightarrow e$ in the $w^{*}$-topology. Thus $a f_{\beta} \rightarrow a$ weakly for every $a \in A$; 
that is, $\left(f_{\beta}\right)_{\beta}$ is a weak approximate identity. Then there exists another net $\left(e_{\alpha}\right)_{\alpha}$, each $e_{\alpha}$ being a convex combination of $f_{\beta}$ 's, which forms an approximate identity in norm [6, Proposition 2.9.14]. Since $\left\|f_{\beta}\right\| \leq\|e\|$ for all $\beta$, $\left\|e_{\alpha}\right\| \leq\|e\| \leq \beta(A)$.

It only remains to show (iii) $\Rightarrow$ (i). If $A$ has a bounded approximate identity, then $A^{* *}$ has a right identity $e$ and the mapping $m \rightarrow e m$ is a bounded projection in $A^{* *}$. This induces a decomposition $A^{* *}=e A^{* *} \oplus(1-e) A^{* *}$. We claim that $j(M(A))=e A^{* *}$. If $T \in M(A)$, then $T^{* *}$ is a multiplier of $A^{* *}$ and hence $T^{* *}(e)=$ $e T^{* *}(e)$.

Conversely, since $A$ is an ideal in $A^{* *}$, each $m \in A^{* *}$ defines a multiplier $T_{m}: a \rightarrow$ $a m$ of $A$, and then it is readily checked that $T_{m}^{* *}(e)=e m$. As $(1-e) A^{* *} \subseteq R\left(A^{* *}\right)$, we conclude that $A^{* *}=j(M(A))+R\left(A^{* *}\right)$. Theorem 2.1 now shows that $A$ is a BSE-algebra.

Of course, our interest is in deciding whether an algebra $A$ is a BSE-algebra. However, a simple consequence of Theorem 3.1 is that, when $A$ is an ideal in its second dual, $A$ cannot possess a $\Delta$-weak bounded approximate identity unless it possesses a bounded approximate identity. This latter condition is usually much easier to check.

Corollary 3.2. Let $A$ be a semisimple commutative BSE-algebra which is an ideal in its second dual. Then $\|a\| \leq \beta(A)\|\widehat{a}\|_{B S E}$ for all $a \in A$. Consequently, if $\beta(A)=1$, then the Fourier-Stieltjes transform $\Gamma: M(A) \rightarrow C^{b}(\Delta(A))$ is a semiembedding.

Proof. By Theorem 3.1, $A$ has a bounded approximate identity $\left(e_{\alpha}\right)_{\alpha}$ bounded by $\beta(A)$. Let $A A^{*}=\left\{a \cdot f: f \in A^{*}, a \in A\right\}$. Since $A$ has a bounded approximate identity, by the module version of Cohen's factorization theorem (see 6. Corollary 2.9.26]) $A A^{*}$ is a closed linear subspace of $A^{*}$. Observe next that $\overline{\operatorname{span}(\Delta(A))}=$ $A A^{*}$. In fact, this can be seen as follows. Let $m \in A^{* *}$ be such that $\langle m, \varphi\rangle=0$ for all $\varphi \in \Delta(A)$. Then

$$
0=\varphi(a)\langle m, \varphi\rangle=\langle m, a \cdot \varphi\rangle=\langle m a, \varphi\rangle
$$

for all $a \in A$ and $\varphi \in \Delta(A)$. Since $A$ is semisimple and an ideal in $A^{* *}$, it follows that $m A=\{0\}$. This in turn implies that $0=\langle m a, f\rangle=\langle m, a \cdot f\rangle$ for all $a \in A$ and $f \in A^{*}$. Thus $m$ annihilates $A A^{*}$ and hence $\overline{\operatorname{span}(\Delta(A))}=A A^{*}$ by the HahnBanach theorem.

Now fix $a \in A$ and let $x \in A_{1}, f \in A_{1}^{*}$ and $\epsilon>0$. Then there exists a functional $\sum_{j=1}^{n} c_{j} \varphi_{j} \in \operatorname{span}(\Delta(A))$ such that $\left\|x \cdot f-\sum_{j=1}^{n} c_{j} \varphi_{j}\right\|_{A^{*}}<\epsilon$. In particular,

$$
\left\|\sum_{j=1}^{n} c_{j} \varphi_{j}\right\|_{A^{*}} \leq\|x\| \cdot\|f\|_{A^{*}}+\epsilon \leq 1+\epsilon .
$$

It follows that

$$
\begin{aligned}
|\langle a, x \cdot f\rangle| & =\left|\left\langle a, x \cdot f-\sum_{j=1}^{n} c_{j} \varphi_{j}\right\rangle\right|+\left|\left\langle a, \sum_{j=1}^{n} c_{j} \varphi_{j}\right\rangle\right| \\
& \leq \epsilon\|a\|+\frac{1}{1+\epsilon} \cdot\|\widehat{a}\|_{B S E} .
\end{aligned}
$$


Letting $\epsilon \rightarrow 0$, we conclude that $|\langle a x, f\rangle| \leq\|\widehat{a}\|_{B S E}$. Since $f \in A_{1}^{*}$ was arbitrary, $\|a x\| \leq\|\widehat{a}\|_{B S E}$. Replacing $x$ by $\frac{1}{\beta(A)} e_{\alpha}$, we get

$$
\|a\|=\lim _{\alpha}\left\|a e_{\alpha}\right\|=\beta(A) \cdot \lim _{\alpha}\left\|a\left(\frac{1}{\beta(A)} e_{\alpha}\right)\right\| \leq \beta(A) \cdot\|\widehat{a}\|_{B S E},
$$

as required. Finally, if $\beta(A)=1$, then $\|a\|=\|\widehat{a}\|_{B S E}$ for all $a \in A$ and hence $\Gamma: M(A) \rightarrow C^{b}(\Delta(A))$ is a semi-embedding by Theorem 2.6.

The following corollary is an immediate consequence of Theorem 3.1. In this context the reader should compare [34, Theorem 8].

Corollary 3.3. Let $A$ be a semisimple commutative Banach algebra which is an ideal in $A^{* *}$. Suppose that $A$ is a BSE-algebra and that $I$ is a closed ideal in $A$. Then

(i) $A / I$ is a BSE-algebra.

(ii) I is a BSE-algebra if and only if I has a bounded approximate identity.

Example 3.4. (1) Let $A=l^{p}, 1<p<\infty$, be the sequence space. Under pointwise multiplication, $A$ is a commutative semisimple Banach algebra. Being reflexive, $A$ is an ideal in its second dual. $A$ does not have a bounded approximate identity and hence fails to be a BSE-algebra.

(2) Let $G$ be an infinite compact abelian group and $\widehat{G}$ the discrete dual group of $G$. For $1 \leq p<\infty, L^{p}(G)$ equipped with convolution is a semisimple commutative Banach algebra with $\Delta\left(L^{p}(G)\right)=\widehat{G}$. Whereas $L^{1}(G)$ is a BSE-algebra, $L^{p}(G)$, for $p>1$, does not have a bounded approximate identity and therefore is not a BSE-algebra.

Theorem 3.1 shows that $l^{p}$ and $L^{p}(G), p>1$, do not even have $\Delta$-weak bounded approximate identities.

The following remark gives a fairly exhaustive class of commutative Banach algebras which are ideals in their second duals. We remind the reader that a commutative Banach algebra is said to be Tauberian if the set of all elements $a \in A$ such that $\widehat{a}$ has compact support in $\Delta(A)$ is dense in $A$.

Remark 3.5. Let $A$ be a semisimple commutative Banach algebra and suppose that $A$ is Tauberian and $\Delta(A)$ is discrete. Then $A$ is an ideal in $A^{* *}$. In fact, for every $a \in A$ such that $\widehat{a}$ has finite support, the multiplication operator $L_{a}: x \rightarrow a x$ is a finite rank operator on $A$, and since such elements are dense in $A$, it follows that $L_{a}$ is compact for every $a \in A$. This property implies that $A$ is an ideal in $A^{* *}$ (compare [29, Proposition 1.4.13]).

\section{The BSE-property: $A$ versus $M(A)$ And $A_{e}$}

In this section we study the problem of how the BSE-property of $A$ is related to the BSE-property of the unitization $A_{e}$ and of the multiplier algebra $M(A)$. We start with $M(A)$.

Theorem 4.1. Let $A$ be a semisimple commutative Banach algebra with bounded approximate identity and satisfying $\|\widehat{a}\|_{B S E}=\|a\|$ for all $a \in A$. If $M(A)$ is a $B S E$-algebra, then so is $A$. 
Proof. Suppose that $M(A)$ is a BSE-algebra. To prove that $A$ is a BSE-algebra we are going to show that the Fourier-Stieltjes transform $\Gamma: M(A) \rightarrow C^{b}(\Delta(A))$ is a semi-embedding. To this end, let $f$ be a continuous function on $\Delta(A)$ belonging to the closure of $\Gamma\left(M(A)_{1}\right)$ in $C^{b}(\Delta(A))$. So there exists a sequence $\left(T_{n}\right)_{n}$ in $M(A)_{1}$ such that $\widehat{T_{n}} \rightarrow f$ uniformly on $\Delta(A)$. For any $a \in A$, we then have

$$
\left\|\widehat{T_{n}} \widehat{a}-f \widehat{a}\right\|_{C^{b}(\Delta(A))}=\left\|\widehat{T_{n} \circ L_{a}}-f \widehat{a}\right\|_{C(\Delta(M(A))}
$$

since $\widehat{a}$ vanishes on $\Delta(M(A)) \backslash \Delta(A)$. Since $M(A)$ is a BSE-algebra, it follows from Theorem 2.4 that $f \widehat{a} \in \widehat{M(A)_{1}}$ for each $a \in A_{1}$. So, for each $a \in A$, there exists $T_{a} \in M(A)$ such that $\widehat{T_{a}}=f \widehat{a}$ on $\Delta(M(A))$. Then, for any $b \in A, f \widehat{a} \widehat{b}=\widehat{T_{a}(b)}$ on $\Delta(A)$. Since $f \widehat{a} \in \widehat{M(A)}$, we see that the product $f \widehat{a} \widehat{b}$ is in $\widehat{A}$. Now, because $A$ has a bounded approximate identity, $A^{2}=A$ by Cohen's factorization theorem. Thus $\widehat{A}^{2}=\widehat{A}$ and so $f \cdot \widehat{A} \subseteq \widehat{A}$. This shows that there a multiplier $T$ of $A$ such that $\widehat{T}=f$ on $\Delta(A)$. Exactly as in the proof of Theorem 2.4 we can now verify that $\|T\| \leq 1$. Thus we have seen that $\Gamma\left(M(A)_{1}\right)$ is closed in $C^{b}(\Delta(A))$, and hence $A$ is a BSE-algebra by Theorem 2.4.

We next aim at results in the reverse direction (Corollaries 4.3 and 4.5).

Proposition 4.2. Let $A$ be a semisimple BSE-algebra with bounded approximate identity. Then the following conditions are equivalent.

(i) $M(A)$ is a BSE-algebra.

(ii) If $\sigma \in C_{B S E}(\Delta(M(A)))$ and $T \in M(A)$ are such that $\sigma=\widehat{T}$ on $\Delta(A)$, then $\sigma=\widehat{T}$ on $\Delta(M(A))$.

Proof. We first remark that if $S, T \in M(A)$ are such that $\widehat{S}=\widehat{T}$ on $\Delta(A)$, then $S=T$. Indeed, this follows immediately from the semisimplicity of $A$.

If (i) holds and $\sigma$ and $T$ are as in (ii), then $\sigma=\widehat{S}$ on $\Delta(M(A))$ for some $S \in M(A)$ and then $\widehat{T}=\widehat{S}=\sigma$ on $\Delta(M(A))$ by the preceding remark. So (i) $\Rightarrow$ (ii).

For (ii) $\Rightarrow$ (i), let $\sigma \in C_{B S E}(\Delta(M(A)))$. Then $\left.\sigma\right|_{\Delta(A)} \in C_{B S E}(\Delta(A))$ by Lemma 1.4 and hence, since $A$ is a BSE-algebra, $\left.\sigma\right|_{\Delta(A)}=\widehat{T}$ for some $T \in M(A)$. By hypothesis, $\sigma=\widehat{T}$ on all of $\Delta(M(A))$. Thus $C_{B S E}(\Delta(M(A))) \subseteq \widehat{M(A)}$ and hence $M(A)$ is a BSE-algebra.

Corollary 4.3. Let $A$ be a semisimple commutative Banach algebra with bounded approximate identity such that $\Delta(A)$ is dense in $\Delta(M(A))$. If $A$ is a BSE-algebra, then so is $M(A)$.

Proof. This is an immediate consequence of the implication (ii) $\Rightarrow$ (i) of Proposition 4.2 .

The next lemma might be known. However, since we are not aware of a reference, we include the simple proof.

Lemma 4.4. Let $A$ be a semisimple commutative Banach algebra with bounded approximate identity. Then $\Delta(A)$ is dense in $\Delta(M(A))$ in the hull-kernel topology. In particular, if $M(A)$ is regular, then $\Delta(A)$ is dense in $\Delta(M(A))$ in the Gelfand topology. 
Proof. Suppose that $T \in M(A)$ is such that $\widehat{T}(\varphi)=0$ for all $\varphi \in \Delta(A)$. Then $\widehat{T(a)}(\varphi)=\widehat{T}(\varphi) \widehat{a}(\varphi)=0$ for all $\varphi \in \Delta(A)$ and $a \in A$. Since $A$ is semisimple, this implies that $T(a)=0$ for all $a \in A$, as required. Because the hull-kernel and the Gelfand topology on $\Delta(M(A))$ coincide if $M(A)$ is regular, the second assertion follows.

As an immediate consequence of Lemma 4.4 and Corollary 4.3 we note

Corollary 4.5. Let $A$ be a semisimple commutative Banach algebra with bounded approximate identity. If $M(A)$ is regular and $A$ is a BSE-algebra, then $M(A)$ is a BSE-algebra.

Now we turn to the problem of whether the unitization of a BSE-algebra also is a BSE-algebra.

Lemma 4.6. Let $A$ be a non-unital commutative Banach algebra and $A_{e}$ its unitization.

(i) If $\sigma \in C_{B S E}\left(\Delta\left(A_{e}\right)\right)$, then $\left.\sigma\right|_{\Delta(A)}-\sigma\left(\varphi_{\infty}\right) \in C_{B S E}(\Delta(A)) \cap C_{0}(\Delta(A))$ and

$$
\left\|\left.\sigma\right|_{\Delta(A)}-\sigma\left(\varphi_{\infty}\right)\right\|_{B S E} \leq\|\sigma\|_{B S E}+\left|\sigma\left(\varphi_{\infty}\right)\right| .
$$

(ii) Let $\tau \in C_{B S E}(\Delta(A)) \cap C_{0}(\Delta(A))$ and $\lambda \in \mathbb{C}$. Define $\sigma$ on $\Delta\left(A_{e}\right)$ by $\sigma(\varphi)=\tau(\varphi)$ for $\varphi \in \Delta(A)$ and $\sigma\left(\varphi_{\infty}\right)=\lambda$. Then $\sigma \in C_{B S E}\left(\Delta\left(A_{e}\right)\right)$ and

$$
\|\sigma\|_{B S E} \leq\|\tau\|_{B S E}+|\lambda| \text {. }
$$

Proof. We apply the characterization of BSE-functions established in Theorem 4 of [34].

(i) There exists a net $\left(x_{\alpha}\right)_{\alpha}$ in $A_{e}$ such that $\left\|x_{\alpha}\right\| \leq\|\sigma\|_{B S E}$ for all $\alpha$ and $\psi\left(x_{\alpha}\right) \rightarrow \sigma(\psi)$ for all $\psi \in \Delta\left(A_{e}\right)$. Let $x_{\alpha}=a_{\alpha}+\lambda_{\alpha} e$, where $a_{\alpha} \in A$ and $\lambda_{\alpha} \in \mathbb{C}$. Then $\lambda_{\alpha}=\varphi_{\infty}\left(x_{\alpha}\right) \rightarrow \sigma\left(\varphi_{\infty}\right)$ and

$$
\widehat{a_{\alpha}}(\varphi)=\widehat{x_{\alpha}}(\varphi)-\lambda_{\alpha} \rightarrow \sigma(\varphi)-\sigma\left(\varphi_{\infty}\right)
$$

for all $\varphi \in \Delta(A)$. Since $\left\|a_{\alpha}\right\| \leq\left\|x_{\alpha}\right\|+\left|\lambda_{\alpha}\right|$ and $\left|\lambda_{\alpha}\right| \rightarrow\left|\sigma\left(\varphi_{\infty}\right)\right|$, it follows that $\left.\sigma\right|_{\Delta(A)}-\sigma\left(\varphi_{\infty}\right) \in C_{B S E}(\Delta(A)) \cap C_{0}(\Delta(A))$ and

$$
\left\|\left.\sigma\right|_{\Delta(A)}-\sigma\left(\varphi_{\infty}\right)\right\|_{B S E} \leq\|\sigma\|_{B S E}+\left|\sigma\left(\varphi_{\infty}\right)\right| .
$$

Clearly, $\left.\sigma\right|_{\Delta(A)}-\sigma\left(\varphi_{\infty}\right)$ vanishes at infinity on $\Delta(A)$.

(ii) Since $\tau \in C_{0}(\Delta(A)), \sigma$ is continuous on $\Delta\left(A_{e}\right)$. There is a bounded net $\left(a_{\alpha}\right)_{\alpha}$ in $A$ such that $\left\|a_{\alpha}\right\| \leq\|\tau\|_{B S E}$ for all $\alpha$ and $\widehat{a_{\alpha}}(\varphi) \rightarrow \tau(\varphi)$ for all $\varphi \in \Delta(A)$. The net $\left(x_{\alpha}\right)_{\alpha} \subseteq A_{e}$ with $x_{\alpha}=a_{\alpha}+\lambda e$, is bounded and satisfies

$$
\widehat{x_{\alpha}}(\varphi)=\widehat{a_{\alpha}}(\varphi)+\lambda \rightarrow \tau(\varphi)+\lambda=\sigma(\varphi)
$$

for all $\varphi \in \Delta(A)$ and $\widehat{x_{\alpha}}\left(\varphi_{\infty}\right)=\lambda \sigma\left(\varphi_{\infty}\right)$. So $\sigma \in C_{B S E}\left(\Delta\left(A_{e}\right)\right)$ and $\|\sigma\|_{B S E} \leq$ $\|\tau\|_{B S E}+|\lambda|$.

Corollary 4.7. Let $A$ be a non-unital commutative Banach algebra and suppose that $A$ has a $\Delta$-weak bounded approximate identity. Then the map $\left.\sigma \rightarrow \sigma\right|_{\Delta(A)}$ is a topological isomorphism from $C_{B S E}\left(\Delta\left(A_{e}\right)\right)$ onto the closed subalgebra

$$
\left(C_{B S E}(\Delta(A)) \cap C_{0}(\Delta(A))\right)+\mathbb{C} 1_{\Delta(A)}
$$

of $C_{B S E}(\Delta(A))$. 
Proof. Let $B=\left(C_{B S E}(\Delta(A)) \cap C_{0}(\Delta(A))\right)+\mathbb{C}_{\Delta(A)}$ and note first that $B \subseteq$ $C_{B S E}(\Delta(A))$ since $A$ has a $\Delta$-weak bounded approximate identity. By Lemma 4.6(i), $\left.\sigma \rightarrow \sigma\right|_{\Delta(A)}-\sigma\left(\varphi_{\infty}\right)$ is a continuous linear mapping of $C_{B S E}\left(\Delta\left(A_{e}\right)\right)$ into $C_{B S E}(\Delta(A)) \cap C_{0}(\Delta(A))$. Consequently, the restriction map $r:\left.\sigma \rightarrow \sigma\right|_{\Delta(A)}$ is a continuous linear mapping from $C_{B S E}\left(\Delta\left(A_{e}\right)\right)$ into $B$. Clearly, $r$ is injective since $\Delta(A)$ is dense in $\Delta\left(A_{e}\right)$. Since both $C_{B S E}\left(\Delta\left(A_{e}\right)\right)$ and $B$ are semisimple, $r$ is a topological isomorphism provided that $r$ is surjective. However, surjectivity follows from Lemma 4.6(ii).

Theorem 4.8. Let $A$ be a non-unital commutative Banach algebra. Then $A_{e}$ is a $B S E$-algebra if and only if

$$
C_{B S E}(\Delta(A)) \cap C_{0}(\Delta(A))=\widehat{A} .
$$

Proof. Suppose that $A_{e}$ is a BSE-algebra and let $\tau \in C_{B S E}(\Delta(A)) \cap C_{0}(\Delta(A))$. Let $\sigma: \Delta\left(A_{e}\right) \rightarrow \mathbb{C}$ be defined as in Lemma 4.6(ii) (taking $\lambda=0$ ). Since $A_{e}$ is a BSE-algebra, $\sigma=\widehat{a+\mu e}$ for some $a \in A$ and $\mu \in \mathbb{C}$. Then $\mu=0$ since $\widehat{a}\left(\varphi_{\infty}\right)=\sigma\left(\varphi_{\infty}\right)=0$, and this shows that $\tau=\widehat{a}$. So $C_{B S E}(\Delta(A)) \cap C_{0}(\Delta(A)) \subseteq \widehat{A}$, and the reverse inclusion is trivial.

Conversely, let $C_{B S E}(\Delta(A)) \cap C_{0}(\Delta(A))=\widehat{A}$. Then, by Lemma 4.6(i), for every $\sigma \in C_{B S E}\left(\Delta\left(A_{e}\right)\right),\left.\sigma\right|_{\Delta(A)}-\sigma\left(\varphi_{\infty}\right) \in \widehat{A}$ and hence $\sigma=\widehat{x}$, where $x=a+\sigma\left(\varphi_{\infty}\right) e \in$ $A_{e}$. Thus $C_{B S E}\left(\Delta\left(A_{e}\right)\right) \subseteq \widehat{A_{e}}$, and again the reverse inclusion is clear.

Corollary 4.9. Suppose that $A$ is Tauberian and has a $\Delta$-weak bounded approximate identity. If $A_{e}$ is a BSE-algebra, then so is $A$.

Proof. Let $C_{B S E}^{0}(\Delta(A))$ be the subalgebra of $C_{B S E}(\Delta(A))$ introduced in [19, Definition 3.5]. Then $C_{B S E}^{0}(\Delta(A)) \subseteq C_{0}(\Delta(A))$ and $\widehat{A} \subseteq C_{B S E}^{0}(\Delta(A))$ whenever $A$ is Tauberian [19, Proposition 4.1]. Hence, if $A_{e}$ is BSE and $A$ is Tauberian, then $\widehat{A}=C_{B S E}^{0}(\Delta(A))$ by (i). This implies that $C_{B S E}(\Delta(A)) \subseteq \widehat{M(A)}$ [19, Proposition 4.3]. On the other hand, $\widehat{M(A)} \subseteq C_{B S E}(\Delta(A))$ since $A$ has a $\Delta$-weak bounded approximate identity [34, Corollary 5]. So $A$ is a BSE-algebra.

The following example shows that it is not true that conversely $A_{e}$ is a BSEalgebra whenever $A$ is a BSE-algebra and $A$ is Tauberian and has a $\Delta$-weak bounded approximate identity. A further application of Theorem 4.8 involving Fourier and Fourier-Stieltjes algebras will be given in the next section.

Example 4.10. Let $A=l^{p}$, as in Example 2. Then $A$ fails to be a BSE-algebra. However, its unitization $A_{e}$ is a BSE-algebra. In fact, since $A_{e}$ is reflexive, $C\left(A_{e}^{* *}\right)=$ $A_{e}^{* *}=A_{e}$, so that $A_{e}$ is a BSE-algebra.

In Section 6 we shall present a second example of a BSE-algebra $A$, for which the unitization fails to be a BSE-algebra.

\section{Fourier and Fourier-Stieltjes Algebras OF LOCALLY COMPACT GROUPS}

The Fourier-Stieltjes algebra, $B(G)$, and the Fourier algebra, $A(G)$, of a locally compact group $G$ have been introduced by Eymard [8]. $B(G)$ consists of all finite linear combinations of continuous positive definite functions on $G$ and is the dual 
space of the group $C^{*}$-algebra of $G$. More precisely, given $u \in B(G)$, there exist a unitary representation $\pi$ of $G$ and $\xi, \eta \in \mathcal{H}(\pi)$, the Hilbert space of $\pi$, such that

$$
u(x)=\langle\pi(x) \xi, \eta\rangle, x \in G \text {, and }\|u\|=\|\xi\| \cdot\|\eta\| .
$$

The Fourier algebra is the closed ideal of $B(G)$ generated by all compactly supported functions in $B(G)$ and turns out to be just the set of coordinate functions of the left regular representation of $G$ in $L^{2}(G)$. Recall that when $G$ is abelian, $B(G)$ is isometrically isomorphic (by means of the Fourier-Stieltjes transform) to $M(\widehat{G})$, the measure algebra of the dual group $\widehat{G}$ of $G$, and this isomorphism identifies $A(G)$ with $L^{1}(\widehat{G})$, the $L^{1}$-algebra of $\widehat{G}$.

We remind the reader that a locally compact group $G$ is amenable if there exists a translation-invariant mean on $L^{\infty}(G)$. Amenability can be characterized in many different ways and plays an important role in harmonic analysis and many other branches of mathematics. Abelian groups and compact groups are amenable and so are closed subgroups and quotient groups of amenable groups. Furthermore, the class of amenable groups is closed under the formation of extensions, and consequently solvable groups are amenable. On the other hand, free groups on more than one generator and non-compact semisimple Lie groups fail to be amenable. As references to amenability of locally compact groups, we mention [15] and 30.

The spectrum of $A(G)$ can be canonically identified with $G$. In fact, the map $x \rightarrow \varphi_{x}$, where $\varphi_{x}(u)=u(x)$ for $u \in A(G)$, is a homeomorphism from $G$ onto $\Delta(A(G))$. Moreover, $A(G)$ is regular in the sense that given a compact subset $C$ of $G$ and a closed subset $E$ of $G$ such that $C \cap E=\emptyset$, there exists $u \in A(G) \cap C_{c}(G)$ with $u(x)=1$ for all $x \in C$ and $u(x)=0$ for all $x \in E$. All these basic results can be found in [8].

Since $A(G)$ is an ideal in $B(G)$, every $u \in B(G)$ defines a multiplier $M_{u}$ of $A(G), M_{u}(v)=u v$ for $v \in A(G)$, and $\left\|M_{u}\right\|=\|u\|$. If $G$ is amenable, each multiplier of $A(G)$ is of this form (see [30. Corollary 19.2]), that is, $M(A(G))=$ $B(G)$. Conversely, as was shown in [28] for discrete groups and in 26] for nondiscrete locally compact groups, the condition that $M(A(G))=B(G)$ implies that $G$ is amenable.

We start with a characterization of those locally compact groups $G$ for which the Fourier algebra $A(G)$ is a BSE-algebra. In the sequel we identify throughout $\Delta(A(G))$ with $G$.

Theorem 5.1. Let $G$ be a locally compact group. Then the following conditions are equivalent.

(i) The Fourier algebra $A(G)$ is a BSE-algebra.

(ii) $A(G)$ has a $\Delta$-weak bounded approximate identity.

(iii) $G$ is amenable.

In this case, the BSE-norm and the $B(G)$-norm on $B(G)=C_{B S E}(G)$ coincide.

Proof. Assume first that $G$ is amenable. Let $\rho$ denote the left regular representation of $G$ and $V N(G)$ the von Neumann algebra generated by the set of operators $\rho(x)$ on $L^{2}(G), x \in G$. Then $V N(G)$ is isometrically isomorphic to the dual space $A(G)^{*}$, and for $u \in A(G)$ and $x \in G$, we have $\langle\rho(x), u\rangle=u(x)$. By [8, Chapitre 2, especially Lemme 2.13 and Corollaire 2.24], a continuous function $u$ on $G$ belongs 
to $B(G)$ if and only if there exists a constant $C \geq 0$ such that

$$
\left|\sum_{j=1}^{n} c_{j} u\left(x_{j}\right)\right| \leq C \cdot\left\|\rho\left(\sum_{j=1}^{n} c_{j} \delta_{x_{j}}\right)\right\|
$$

for any choice of finitely many elements $x_{1}, \ldots, x_{n}$ of $G$ and complex numbers $c_{1}, \ldots, c_{n}$. In this case, $\|u\|$ equals the infimum of all such constants $C$. On the other hand, by definition of the BSE-norm,

$$
\|u\|_{B S E}=\sup \left\{\left|\sum_{j=1}^{n} c_{j} u\left(x_{j}\right)\right|:\left\|\sum_{j=1}^{n} c_{j} \varphi_{x_{j}}\right\|_{A(G)^{*}} \leq 1\right\} .
$$

Since

$$
\begin{aligned}
\left\|\sum_{j=1}^{n} c_{j} \varphi_{x_{j}}\right\|_{A(G)^{*}} & =\sup \left\{\left|\sum_{j=1}^{n} c_{j} v\left(x_{j}\right)\right|: v \in A(G),\|v\| \leq 1\right\} \\
& =\sup \left\{\left|\left\langle\rho\left(\sum_{j=1}^{n} c_{j} \delta_{x_{j}}\right), v\right\rangle\right|: v \in A(G),\|v\| \leq 1\right\} \\
& =\left\|\rho\left(\sum_{j=1}^{n} c_{j} \delta_{x_{j}}\right)\right\|,
\end{aligned}
$$

it follows that $C_{B S E}(G)=B(G)$ and $\|u\|_{B S E}=\|u\|$ for each $u \in B(G)$.

Since a semisimple commutative Banach algebra has a $\Delta$-weak bounded approximate identity if (and only if) $\widehat{M(A)} \subseteq C_{B S E}(\Delta(A)$ ), (i) $\Rightarrow$ (ii). It therefore only remains to show (ii) $\Rightarrow$ (iii). So let $\left(e_{\alpha}\right)_{\alpha}$ be a $\Delta$-weak bounded approximate identity and let $e \in A(G)^{* *}$ be a $w^{*}$-cluster point of $\left(e_{\alpha}\right)_{\alpha}$. Then $\langle e, \varphi\rangle=1$ for all $\varphi \in \Delta(A(G))=G$ and hence, by [38, Proposition 2.8], $\Delta(A(G))$ is weakly closed in $A(G)^{*}$. This in turn implies that $G$ is amenable [4, Corollary 2.8].

Theorem 5.1, (iii) $\Rightarrow$ (i), combined with Theorem 2.4 reproves the following known result [8, Corollaire 2.25]. Note that the Fourier-Stieltjes transform $\Gamma$ : $B(G) \rightarrow C^{b}(G)$ is just the identity mapping.

Corollary 5.2. Let $G$ be an amenable locally compact group. Then the unit ball $B(G)_{1}$ of $B(G)$ is norm closed in $C^{b}(G)$.

Let $A$ be a commutative Banach algebra and $E$ a closed subset of $\Delta(A)$. We remind the reader that associated to $E$ are two distinguished ideals, namely,

$$
I(E)=\left\{a \in A:\left.\widehat{a}\right|_{E}=0\right\}
$$

and

$$
j(E)=\{a \in A: \widehat{a} \text { has compact support disjoint from } E\} .
$$

If $A$ is semisimple and regular, then $I(E)$ is the largest ideal of $A$ with hull $E$ and $j(E)$ is the smallest such ideal. Then $E$ is called a set of synthesis if $I(E)=\overline{j(E)}$.

For any (discrete) group $D$, the coset ring $\mathcal{R}(D)$ is defined to be the Boolean ring of subsets of $D$ generated by all left cosets of subgroups of $D$. If $G$ is a locally 
compact group and $G_{d}$ denotes the group $G$ with the discrete topology, then the closed coset ring of $G, \mathcal{R}_{c}(G)$, is defined to be

$$
\mathcal{R}_{c}(G)=\left\{E \in \mathcal{R}\left(G_{d}\right): E \text { is closed in } G\right\} .
$$

Then $\mathcal{R}_{c}(G)$ is precisely the collection of all subsets $E$ of $G$ of the form

$$
E=\bigcup_{i=1}^{n}\left(a_{i} H_{i} \backslash \bigcup_{j=1}^{m_{i}} b_{i j} K_{i j}\right),
$$

where $a_{i}, b_{i j} \in G, H_{i}$ is a closed subgroup of $G$, and $K_{i j}$ is an open subgroup of $H_{i}, n, m_{i} \in \mathbb{N}_{0}, 1 \leq i \leq n, 1 \leq j \leq m_{i}$ [11.

Let $G$ be a locally compact abelian group, and for any closed subset $E$ of $\widehat{G}=$ $\Delta\left(L^{1}(G)\right)$, let $I(E)=\left\{f \in L^{1}(G): \widehat{f}=0\right.$ on $\left.E\right\}$. It was recently shown in [19. Theorem 5.6] that the ideal $I(E)$ of $L^{1}(G)$ is a BSE-algebra if and only if $E \in \mathcal{R}_{c}(\widehat{G})$. The following result is a far-reaching generalization of Theorem 5.6 of [19. Note that by [13, Theorem 2.3] the ideals in (iii) of the following theorem are precisely the closed ideals in $A(G)$ which possess bounded approximate identities.

It was shown in [12, Theorem 1 ] that if $G$ is any locally compact group and $I$ is a closed ideal in $A(G)$, which has a $\Delta$-weak bounded approximate identity, then the hull of $I$ is an element of $\mathcal{R}_{c}(G)$. The following theorem improves [12, Corollary 2]. However, the proof of the implication (ii) $\Rightarrow$ (iii) follows the arguments used in the proof of [12, Theorem 1] and is only included for the reader's convenience.

Theorem 5.3. Let $G$ be an amenable locally compact group and I a closed ideal of the Fourier algebra $A(G)$. Then the following are equivalent.

(i) $I$ is a BSE-algebra.

(ii) I has a $\Delta$-weak bounded approximate identity.

(iii) $I$ is of the form $I=I(E)=\left\{u \in A(G):\left.u\right|_{E}=0\right\}$ for some set $E$ in $\mathcal{R}_{c}(G)$.

Proof. If $E \in \mathcal{R}_{c}(G)$, then $I(E)$ is a BSE-algebra by Corollary 2.9 of 22 .

Therefore we only have to show (ii) $\Rightarrow$ (iii). Thus let $I$ be a closed ideal of $A(G)$ which has a $\Delta$-weak bounded approximate identity, $\left(u_{\alpha}\right)_{\alpha}$ say. Let

$$
E=\{x \in G: u(x)=0 \text { for all } u \in I\} .
$$

It follows that $u_{\alpha}(x)=0$ for all $x \in E$ and $u_{\alpha}(x) \rightarrow 1$ for all $x \in G \backslash E$.

Consider the functions $u_{\alpha}$ as functions on $G_{d}$. Then $\left(u_{\alpha}\right)_{\alpha}$ is a bounded net in $B\left(G_{d}\right)=C^{*}\left(G_{d}\right)^{*}$. Let $u$ be a $w^{*}$-cluster point of $\left(u_{\alpha}\right)_{\alpha}$ in $B\left(G_{d}\right)$. Then, denoting again by $\left(u_{\alpha}\right)_{\alpha}$ the corresponding subnet,

$$
u_{\alpha}(x)=\left\langle u_{\alpha}, \delta_{x}\right\rangle \rightarrow\left\langle u, \delta_{x}\right\rangle=u(x)
$$

for every $x \in G$ and hence $u(x)=1$ for all $x \in G \backslash E$ and $u(x)=0$ for all $x \in E$. Host's [17 idempotent theorem now implies that $E \in \mathcal{R}\left(G_{d}\right)$. Since $E$ is closed in $G, E \in \mathcal{R}_{c}(G)$. Now, since $G$ is amenable, every set in $\mathcal{R}_{c}(G)$ is a set of synthesis for $A(G)$ [13, Lemma 2.2] and therefore $I=I(E)$, as was to be shown.

Let $G$ be a locally compact abelian group. The Bochner-Schoenberg-Eberlein theorem characterizes the elements of $M(G)$ in terms of their Fourier-Stieltjes transforms among all bounded continuous functions on $\widehat{G}$. A theorem of Doss [7] singles out among these functions those which are Fourier transforms of elements of $L^{1}(G)$. This latter result was generalized to $A(G)$ for an amenable locally compact group $G$ 
in [10]. In [19, the authors have recently taken up for the first time the problem of characterizing the image of $A$ under the Gelfand homomorphism within $C_{0}(\Delta(A))$ for general commutative Banach algebras and for ideals in $L^{1}(G)$ in particular. We next study this question for ideals in the Fourier algebra $A(G)$.

Theorem 5.4. Let $G$ be a locally compact group and $E$ a closed subset of $G=$ $\Delta(A(G))$. For $\sigma \in C_{B S E}(G \backslash E)$, consider the following two conditions:

(i) $\sigma \in I(E)$.

(ii) For each $\epsilon>0$ there exists a compact subset $K$ of $G \backslash E$ such that for any $x_{1}, \ldots, x_{n} \in(G \backslash E) \backslash K$ and $c_{1}, \ldots, c_{n} \in \mathbb{C}$,

$$
\left\|\sum_{j=1}^{n} c_{j} \varphi_{x_{j}}\right\|_{I(E)^{*}} \leq 1 \Rightarrow\left|\sum_{j=1}^{n} c_{j} \sigma\left(x_{j}\right)\right| \leq \epsilon .
$$

Then (i) $\Rightarrow$ (ii) provided that $E$ is a set of synthesis, and conversely (ii) $\Rightarrow$ (i) if $G$ is amenable.

Proof. Suppose first that $\sigma=u \in I(E)$ and that $E$ is a set of synthesis. Given $\epsilon>0$, there exists $v \in A(G)$ such that $v$ has compact support disjoint from $E$ and $\|u-v\|_{A(G)} \leq \epsilon$. Now, for any $x_{1}, \ldots, x_{n} \in(G \backslash E) \backslash \operatorname{supp} v$ and $c_{1}, \ldots, c_{n} \in \mathbb{C}$,

$$
\begin{aligned}
\left|\sum_{j=1}^{n} c_{j} \sigma\left(x_{j}\right)\right| & =\left|\left\langle\sum_{j=1}^{n} c_{j} \varphi_{x_{j}}, u\right\rangle-\left\langle\sum_{j=1}^{n} c_{j} \varphi_{x_{j}}, v\right\rangle\right| \\
& \leq\left\|\sum_{j=1}^{n} c_{j} \varphi_{x_{j}}\right\|_{I(E)^{*}} \cdot\|u-v\|_{A(G)},
\end{aligned}
$$

and hence $\left|\sum_{j=1}^{n} c_{j} \sigma\left(x_{j}\right)\right| \leq \epsilon$ whenever $\left\|\sum_{j=1}^{n} c_{j} \varphi_{x_{j}}\right\|_{I(E)^{*}} \leq 1$. So (ii) holds for $\sigma$.

Conversely, suppose that $\sigma$ satisfies (ii) and that $G$ is amenable. Then $\sigma$ vanishes at infinity on $G \backslash E$ and hence the extension $\widetilde{\sigma}: G \rightarrow \mathbb{C}$ defined by $\widetilde{\sigma}(x)=0$ for $x \in E$ belongs to $C_{B S E}(G)$ by Lemma 1.5(i). Let $\epsilon>0$ be given and let $K$ be a compact subset of $G \backslash E$ as in (ii). Let $x_{1}, \ldots, x_{n} \in G \backslash K$ and $c_{1}, \ldots, c_{n} \in \mathbb{C}$. Then we can assume that $x_{j} \in G \backslash E$ exactly for $j \leq m$ for some $0 \leq m \leq n$. Then

$$
\sum_{j=1}^{n} c_{j} \widetilde{\sigma}\left(x_{j}\right)=\sum_{j=1}^{m} c_{j} \sigma\left(x_{j}\right)
$$

and

$$
\left\|\left.\sum_{j=1}^{m} c_{j} \varphi_{x_{j}}\right|_{I(E)}\right\|_{I(E)^{*}}=\left\|\left.\sum_{j=1}^{n} c_{j} \varphi_{x_{j}}\right|_{I(E)}\right\|_{I(E)^{*}} \leq\left\|\sum_{j=1}^{n} c_{j} \varphi_{x_{j}}\right\|_{A(G)^{*}} .
$$

Thus

$$
\left\|\sum_{j=1}^{n} c_{j} \varphi_{x_{j}}\right\|_{A(G)^{*}} \leq 1 \Rightarrow\left|\sum_{j=1}^{n} c_{j} \widetilde{\sigma}\left(x_{j}\right)\right| \leq \epsilon .
$$

From the result of [10] we conclude that $\widetilde{\sigma}=u$ for some $u \in A(G)$. Since $u(x)=$ $\widetilde{\sigma}(x)=0$ for all $x \in E, u \in I(E)$ and so (i) holds for $\sigma$. 
In the terminology of [19, combining Theorems 5.3 and 5.4 says that if $G$ is an amenable locally compact group and $E \in \mathcal{R}_{c}(G)$, then the ideal $I(E)$ is a BEDalgebra (here BED stands for Bochner-Eberlein-Doss).

Let $H$ be a non-discrete locally compact abelian group. Then the measure algebra $M(H)$ is not BSE. This was pointed out in [34, p. 157] and is a consequence of the fact (which we already exploited in Remark 2.7) that there exists a singular measure $\mu \in M(H)$ such that the Fourier-Stieltjes transform $\widehat{\mu}$ vanishes at infinity on $\widehat{H}$. Consequently, the Fourier-Stieltjes algebra $B(G)$ of a non-compact locally compact abelian group $G$ fails to be a BSE-algebra. We shall use this to draw the same conclusion for a nilpotent locally compact group. We need the following preparatory lemma.

Lemma 5.5. Let $G$ be an amenable locally compact group and $N$ a closed normal subgroup of $G$. If $B(G)$ is a BSE-algebra, then so is $B(G / N)$.

Proof. Let $\sigma \in C_{B S E}(G / N)$ and let $q: G \rightarrow G / N$ denote the quotient homomorphism. Then, for any $x_{1}, \ldots, x_{n} \in G$ and $c_{1}, \ldots, c_{n} \in \mathbb{C}$,

$$
\begin{aligned}
\left|\sum_{j=1}^{n} c_{j}(\sigma \circ q)\left(x_{j}\right)\right| & =\left|\sum_{j=1}^{n} c_{j} \sigma\left(x_{j} N\right)\right| \leq\|\sigma\|_{B S E}\left\|\sum_{j=1}^{n} c_{j} \varphi_{x_{j} N}\right\|_{A(G / N)^{*}} \\
& =\|\sigma\|_{B S E} \cdot \sup \left\{\left|\sum_{j=1}^{n} c_{j} v\left(x_{j} N\right)\right|: v \in A(G / N)_{1}\right\} .
\end{aligned}
$$

Since $G$ is amenable, given any $c>1$, there exists $w \in A(G)$ such that $\|w\| \leq c$ and $w\left(x_{j}\right)=1$ for $j=1, \ldots, n$ (see [28, Lemma 15.21]). Then $u=w(v \circ q) \in A(G)$ and $u$ satisfies $\|u\| \leq c\|v \circ q\|=c\|v\|$ and

$$
\sum_{j=1}^{n} c_{j} v\left(x_{j} N\right)=\sum_{j=1}^{n} c_{j} u\left(x_{j}\right)
$$

It follows that

$$
\left|\sum_{j=1}^{n} c_{j}(\sigma \circ q)\left(x_{j}\right)\right| \leq c\|\sigma\|_{B S E} \cdot\left\|\sum_{j=1}^{n} c_{j} \varphi_{x_{j}}\right\|_{A(G)^{*}} .
$$

So $\sigma \circ q \in C_{B S E}(G)$ and hence, since $M(A(G))=B(G), \sigma \circ q \in B(G)$. This implies that $\sigma \in B(G / N)$, as was to be shown.

Proposition 5.6. Let $G$ be a nilpotent locally compact group. Then $B(G)$ is a $B S E$-algebra (if and) only if $G$ is compact.

Proof. We only have to show that if $B(G)$ is a BSE-algebra, then $G$ must be compact. Let

$$
\{e\}=Z_{0}(G) \subseteq Z_{1}(G)=Z(G) \subseteq \cdots \subseteq Z_{n}(G)=G
$$

denote the ascending central series of $G$; that is, $Z_{j+1}(G) / Z_{j}(G)$ is the centre of $G / Z_{j}(G), j=0, \ldots, n-1$. We prove by induction that $G / Z_{n-j}(G)$ is compact. Suppose that, for some $k, G / Z_{k+1}(G)$ is known to be compact, and consider $H=$ $G / Z_{k}(G)$. Then $B(H)$ is a BSE-algebra by Lemma 5.5 and $H / Z(H)$ is compact 
since $Z(H)=Z_{k+1}(G) / Z_{k}(G)$. Since $H / Z(H)$ is compact, the closed commutator subgroup, $C$ say, of $H$ is compact [16. Now, as $B(H / C)$ is BSE and $H / C$ is abelian, $H / C$ is compact and hence so is $H$. This finishes the induction and shows that $G$ is compact.

A natural question arising is whether $B(G)$ always fails to be a BSE-algebra when $G$ is non-compact. It will turn out shortly that this is not the case, regardless of whether $G$ is amenable (Theorem 5.9 and Example 5.11) or not (Corollary 5.8).

Theorem 5.7. Let $G$ be a locally compact group such that $B(G)=A(G)+\mathbb{C} 1_{G}$. Then $B(G)$ is a BSE-algebra.

Proof. The hypothesis implies that $B(G)$ is the unitization of $A(G)$. Therefore, by Theorem 4.8, we have to verify that $C_{B S E}(G) \cap C_{0}(G)=A(G)$. It is clear that $A(G)$ is contained in $C_{B S E}(G) \cap C_{0}(G)$.

Conversely, let $\sigma \in C_{B S E}(G) \cap C_{0}(G)$. Then $\widetilde{\sigma}$, the extension of $\sigma$ to all of $\Delta(B(G))=G \cup\left\{\varphi_{\infty}\right\}$ defined by $\widetilde{\sigma}\left(\varphi_{\infty}\right)=0$, is in $C_{B S E}(\Delta(B(G))$ (Lemma 1.5(i)). Hence there exists a bounded net $\left(u_{\alpha}\right)_{\alpha}$ in $B(G)$ such that $\widehat{u_{\alpha}}(\varphi) \rightarrow \widetilde{\sigma}(\varphi)$ for all $\varphi \in \Delta(B(G))$. This implies that $u_{\alpha} \in B(G) \cap C_{0}(G)$ and $u_{\alpha}(x) \rightarrow \sigma(x)$ for all $x \in G$ and $u_{\alpha}\left(\varphi_{\infty}\right) \rightarrow 0$.

Now let $G_{d}$ denote the group $G$ with the discrete topology. Since the net $\left(u_{\alpha}\right)_{\alpha}$ is bounded and converges pointwise to $\sigma, \sigma \in B\left(G_{d}\right)$ by [8, Corollaire 2.25]. However, $\sigma \in C_{0}(G)$, and hence $\sigma \in B(G) \cap C_{0}(G)=A(G)$ by [8, Corollaire 2.24]. This finishes the proof.

Corollary 5.8. Let $G$ be a connected simple Lie group with finite centre. Then $B(G)$ is a BSE-algebra.

Proof. If $G$ is compact, nothing has to be shown, whereas if $G$ is non-compact, the hypothesis of the theorem is satisfied by [5, Theorem].

Theorem 5.9. Let $G$ be an amenable locally compact group and let $N$ be a closed normal subgroup of $G$ such that $G / N$ is compact. Suppose that, with $q: G \rightarrow G / N$ denoting the quotient homomorphism,

$$
B(G)=B(G / N) \circ q+A(G) .
$$

Then $G$ is dense in $\Delta(B(G))$ and $B(G)$ is a BSE-algebra.

Proof. Of course, we can assume that $G$, and hence $N$, is non-compact. Since $G$ is amenable, $A(G)$ is a BSE-algebra and $M(A(G))=B(G)$. It suffices to show that $G$ is dense in $\Delta(B(G))$ because then $B(G)$ is a BSE-algebra by Corollary 4.3.

Since $G / N$ is compact and $B(G)=B(G / N) \circ q+A(G)$, the Gelfand space of $B(G)$, as a set, is equal to the disjoint union of $G$ and $\Delta(B(G / N))=G / N$. We have to verify that given $a N \in G / N$ and any neighbourhood $U$ of $a N$ in $\Delta(B(G))$, $U \cap G \neq \emptyset$. Thus, let $\epsilon>0$ and $u_{j}=v_{j} \circ q+w_{j} \in B(G), j=1, \ldots, n$, where $v_{j} \in B(G / N)$ and $w_{j} \in A(G)$, and let

$$
U=U\left(a N, u_{1}, \ldots, u_{n}, \epsilon\right)=U_{1} \cup U_{2},
$$

where $U_{1}=\left\{b N:\left|v_{j}(b N)-v_{j}(a N)\right|<\epsilon, j=1, \ldots, n\right\}$ and

$$
U_{2}=\left\{x \in G:\left|u_{j}(x)-u_{j}(a)\right|<\epsilon, j=1, \ldots, n\right\} .
$$


Since $A(G) \subseteq C_{0}(G)$, there exists a compact subset $C$ of $G$ such that $\left|w_{j}(x)\right|<\epsilon$ for $j=1, \ldots, n$ and all $x \in G \backslash C$. Moreover, since $N$ is non-compact, $a N \cap(G \backslash C) \neq \emptyset$. For any $x \in a N \cap(G \backslash C)$, we then have

$$
\left|u_{j}(x)-u_{j}(a)\right|=\left|v_{j}(x N)-v_{j}(a N)+w_{j}(x)\right|=\left|w_{j}(x)\right|<\epsilon
$$

for all $j$, so that $x \in U_{2}$. Consequently, $G$ is dense in $\Delta(B(G))$.

Corollary 5.10. Let $G$ be an amenable locally compact group and $N$ a closed normal subgroup of $G$ such that $G / N$ is abelian and

$$
B(G)=B(G / N) \circ q+A(G) .
$$

Then the following conditions are equivalent.

(i) $G / N$ is compact.

(ii) $G$ is dense in $\Delta(B(G))$.

(iii) $B(G)$ is a BSE-algebra.

Proof. (i) $\Rightarrow$ (ii) and (ii) $\Rightarrow$ (iii) follow from Theorem 5.9. If (iii) holds, then $B(G / N)$ is a BSE-algebra (Lemma 5.5) and this forces $G / N$ to be compact since $G / N$ is abelian.

As mentioned earlier, if $G$ is a non-compact abelian (more generally, non-compact nilpotent) locally compact group, then $A(G)$ is a BSE-algebra, whereas $B(G)$ is not. On the other hand, for a non-compact connected simple Lie group with finite centre, $B(G)$ is BSE (Corollary 5.8), but $A(G)$ is not BSE. We now exhibit examples of noncompact locally compact groups $G$ satisfying any of the remaining two alternatives: both $A(G)$ and $B(G)$ are BSE-algebras, and none of $A(G)$ and $B(G)$ is a BSEalgebra.

Example 5.11. Let $G$ be a semidirect product $G=N \rtimes K$, where

(i) $K$ is a compact group acting on the locally compact abelian group $N$, with each of the groups second countable, and

(ii) the dual space $\widehat{G}$ of $G$ is countable and decomposes as $\widehat{K} \circ q \cup\left\{\pi_{j}: j \in \mathbb{N}\right\}$, where $q: G \rightarrow K$ is the quotient map and each $\pi_{j}$ is a subrepresentation of the left regular representation of $G$.

For such $G$, it was shown in [32, Proposition 2.1] that

$$
B(G)=A(K) \circ q+A(G) .
$$

Thus, since $G$ is amenable and $G / N$ is compact, $B(G)$ is a BSE-algebra by Theorem 5.9. Of course, $A(G)$ is a BSE-algebra as well. There are several examples satisfying conditions (i) and (ii). We mention two of them, taken from [32] and 25], respectively.

(1) For any prime number $p$, let $\mathbb{Q}_{p}$ denote the locally compact field of $p$-adic numbers, $\mathbb{O}_{p}$ the compact open subring of $p$-adic integers and $\mathbb{T}_{p}$ the multiplicative group of $p$-adic numbers of valuation 1 . Let $S L\left(n, \mathbb{O}_{p}\right)$ denote the multiplicative group of $n \times n$ matrices with entries in $\mathbb{O}_{p}$ and determinant of valuation 1 . This compact group acts on the vector space $\mathbb{Q}_{p}^{n}$ by matrix multiplication and, as shown in [27, the semidirect product $G=\mathbb{Q}_{p}^{n} \rtimes S L\left(n, \mathbb{O}_{p}\right)$ satisfies condition (ii) above.

(2) Let $G$ be the semidirect product $G=N \rtimes K$, where $N$ is a countably infinite discrete abelian group and $K$ is a compact group of automorphisms of $N$ such that $\widehat{N}$ decomposes into $\left\{1_{N}\right\}$ and countably many open $K$-orbits. As shown in [25], one can for instance take $N=\sum_{n=1}^{\infty} \mathbb{Z}_{2}$, the infinite direct sum of copies of $\mathbb{Z}_{2}$, and 
for $K$ the group of all automorphisms of $N$ which can be written as infinite upper triangular $\mathbb{Z}_{2}$-valued matrices.

Note that the group $\mathbb{Q}_{p} \rtimes S L\left(1, \mathbb{O}_{p}\right)=\mathbb{Q}_{p} \rtimes \mathbb{T}_{p}$ in Example 5.10(1) is 2-step solvable. This shows that Proposition 5.6 does not extend to solvable groups.

Example 5.12. Let $\mathbb{F}_{2}$ be the free group on two generators $a$ and $b$, and let $\alpha$ denote the automorphism of $\mathbb{F}_{2}$ interchanging $a$ and $b$. Let $H$ be the cyclic group generated by $\alpha$ and form the semidirect product $G=\mathbb{F}_{2} \rtimes H$. Then $G$ is not amenable since $\mathbb{F}_{2}$ is not amenable, and hence $A(G)$ is not a BSE-algebra. However, $B(G)$ also fails to be a BSE-algebra because $B(H)=B\left(G / \mathbb{F}_{2}\right)$ is not BSE (Lemma 5.5).

We conclude this section by considering two examples concerning the unitization $A(G)_{e}$ of $A(G)$.

Example 5.13. (1) Let $G$ be the $(a x+b)$-group, that is, the semidirect product $G=\mathbb{R} \rtimes \mathbb{R}_{+}^{\times}$, where the multiplicative group of positive real numbers acts on $\mathbb{R}$ by multiplication. As shown in $\left[23, B(G)=B\left(\mathbb{R}_{+}^{\times}\right) \circ q+A(G)\right.$. It follows from Theorem 2.3 that $A(G)_{e}$ is a BSE-algebra. However, by Lemma $5.5, B(G)$ is not BSE since $B(G / \mathbb{R})$ is not BSE.

(2) Let $G$ be a second countable non-compact locally compact group whose regular representation is not completely reducible. Then $A(G) \neq B(G) \cap C_{0}(G)$ [1, 9. Thus, if in addition $G$ is amenable, then $A(G)$ is a BSE-algebra, but $A(G)_{e}$ is not.

\section{Two EXAMPLES}

In this final section we present two examples. The first one shows that the algebra $\operatorname{Lip}_{\alpha} X$ of Lipschitz functions of order $\alpha, 0<\alpha \leq 1$, on a compact metric space $X$ is a BSE-algebra, but the subalgebra $\operatorname{lip}_{\alpha} X$ for $0<\alpha<1$ fails to be a BSE-algebra. The second example concerns the BSE-algebra

$$
A=\left\{f \in \operatorname{Lip}_{1}(\mathbb{R}) \cap C_{0}(\mathbb{R}): \lim _{R \rightarrow \infty} \rho_{R}(f)=0\right\}
$$

(see below for the definition of $\rho_{R}(f)$ ). As an application of Theorem 4.8, we show that its unitization is not a BSE-algebra.

Example 6.1. Let $X$ be a compact metric space with metric $d$ and let $0<\alpha \leq 1$. Then $\operatorname{Lip}_{\alpha} X$ is the space of all continuous complex-valued functions $f$ on $X$ such that

$$
p_{\alpha}(f)=\sup \left\{\frac{|f(x)-f(y)|}{d(x, y)^{\alpha}}: x, y \in X, x \neq y\right\}
$$

is finite. With pointwise multiplication and the norm $\|f\|=\|f\|_{\infty}+p_{\alpha}(f), \operatorname{Lip}_{\alpha} X$ is a commutative Banach algebra. The map $x \rightarrow \varphi_{x}$, where $\varphi_{x}(f)=f(x)$ for $f \in \operatorname{Lip}_{\alpha} X$, is a homeomorphism from $X$ onto $\Delta\left(\operatorname{Lip}_{\alpha} X\right)$ (see [6, Theorem 4.4.24]).

We claim that $\operatorname{Lip}_{\alpha} X$ is a BSE-algebra. To see this, let $\sigma \in C_{B S E}(X)$ and $C=\|\sigma\|_{B S E}$. Then, for any $x, y \in X$ with $x \neq y$,

$$
\begin{aligned}
\frac{|\sigma(x)-\sigma(y)|}{d(x, y)^{\alpha}} & \leq C \cdot \sup \left\{\frac{|f(x)-f(y)|}{d(x, y)^{\alpha}}: f \in \operatorname{Lip}_{\alpha} X,\|f\| \leq 1\right\} \\
& \leq C \cdot \sup \left\{p_{\alpha}(f): f \in \operatorname{Lip}_{\alpha} X,\|f\| \leq 1\right\} \leq C .
\end{aligned}
$$

So $p_{\alpha}(\sigma)<\infty$ and hence $\sigma \in \operatorname{Lip}_{\alpha} X$. 
On $\operatorname{Lip}_{\alpha} X$, the original norm and the BSE-norm coincide. Since $\|f\|_{B S E} \leq\|f\|$, we only have to show the reverse inequality. Let $f \neq 0$ and $0<\epsilon<\|f\|$. There exist $x_{1}, x_{2}, x_{3} \in X$ such that $x_{2} \neq x_{3}$ and

$$
\left|f\left(x_{1}\right)\right|+\frac{\left|f\left(x_{2}\right)-f\left(x_{3}\right)\right|}{d\left(x_{2}, x_{3}\right)^{\alpha}} \geq\|f\|-\epsilon .
$$

Choose $\gamma, \delta \in \mathbb{C}$ of absolute value one such that $\delta\left(f\left(x_{2}\right)-f\left(x_{3}\right)\right)=\left|f\left(x_{2}\right)-f\left(x_{3}\right)\right|$ and $\gamma f\left(x_{1}\right)=\left|f\left(x_{1}\right)\right|$, and set $c_{1}=\gamma, c_{2}=\delta d\left(x_{2}, x_{3}\right)^{-\alpha}$ and $c_{3}=-c_{2}$. Then

$$
\left|\sum_{j=1}^{3} c_{j} f\left(x_{j}\right)\right|=\left|f\left(x_{1}\right)\right|+\frac{\left|f\left(x_{2}\right)-f\left(x_{3}\right)\right|}{d\left(x_{2}, x_{3}\right)^{\alpha}} \geq\|f\|-\epsilon .
$$

On the other hand, for any $g \in \operatorname{Lip}_{\alpha} X$,

$$
\begin{aligned}
\left|\left\langle\sum_{j=1}^{3} c_{j} \varphi_{x_{j}}, g\right\rangle\right| & =\left|\gamma g\left(x_{1}\right)+\delta \frac{g\left(x_{2}\right)-g\left(x_{3}\right)}{d\left(x_{2}, x_{3}\right)^{\alpha}}\right| \\
& \leq\left|g\left(x_{1}\right)\right|+\frac{\left|g\left(x_{2}\right)-g\left(x_{3}\right)\right|}{d\left(x_{2}, x_{3}\right)^{\alpha}} \\
& \leq\|g\|,
\end{aligned}
$$

so that $\left\|\sum_{j=1}^{3} c_{j} \varphi_{x_{j}}\right\| \leq 1$. It follows that $\|f\|_{B S E} \geq\|f\|-\epsilon$. Since $\epsilon>0$ was arbitrary, $\|f\|_{B S E} \geq\|f\|$.

For $0<\alpha<1$, let $\operatorname{lip}_{\alpha} X$ be the subspace of all functions $f \in \operatorname{Lip}_{\alpha} X$ such that

$$
\frac{|f(x)-f(y)|}{d(x, y)^{\alpha}} \rightarrow 0 \quad \text { as } \quad d(x, y) \rightarrow 0 .
$$

Then $\operatorname{lip}_{\alpha} X$ is a closed subalgebra of $\operatorname{Lip}_{\alpha} X$ and the map $\left.x \rightarrow \varphi_{x}\right|_{\operatorname{lip}_{\alpha} X}$ is a homeomorphism from $X$ onto $\Delta\left(\operatorname{lip}_{\alpha} X\right)$. Now, the two algebras $\left(\operatorname{lip}_{\alpha} X\right)^{* *}$ and $\operatorname{Lip}_{\alpha} X$ are isometrically isomorphic [6, Theorem 4.4.34]. Thus, identifying $\Delta\left(\operatorname{lip}_{\alpha} X\right)$ with $X$, we have

$$
C_{B S E}(X)=\left.\left(\operatorname{lip}_{\alpha} X\right)^{* *}\right|_{X} \cap C^{b}(X)=\operatorname{Lip}_{\alpha} X
$$

34, Theorem 4], and hence $\operatorname{lip}_{\alpha} X$ fails to be a BSE-algebra.

Example 6.2. Let $C^{b}(\mathbb{R})$ denote the space of all bounded continuous functions on the real line $\mathbb{R}$, and for each $f \in C^{b}(\mathbb{R})$, let

$$
\rho(f)=\sup \left\{\left|\frac{f(s)-f(t)}{s-t}\right|: s, t \in \mathbb{R}, s \neq t\right\} \in[0, \infty] .
$$

Then $\operatorname{Lip}_{1}(\mathbb{R})=\left\{f \in C^{b}(\mathbb{R}): \rho(f)<\infty\right\}$, equipped with pointwise operations and the norm $\|f\|=\|f\|_{\infty}+\rho(f)$, is a commutative Banach algebra, the algebra of Lipschitz functions of order 1 . Moreover, for any $R>0$, let

$$
\rho_{R}(f)=\sup \left\{\left|\frac{f(s)-f(t)}{s-t}\right|:|s|,|t| \geq R, s \neq t\right\} .
$$

Define a closed subalgebra $A$ of $\operatorname{Lip}_{1}(\mathbb{R})$ by

$$
A=\left\{f \in \operatorname{Lip}_{1}(\mathbb{R}) \cap C_{0}(\mathbb{R}): \lim _{R \rightarrow \infty} \rho_{R}(f)=0\right\} .
$$

Then $A$ is regular and Tauberian, and $\Delta(A)$ can be canonically identified with $\mathbb{R}$ by means of point evaluations. It was shown in [19] that $A$ is a BSE-algebra. More precisely, according to the proof of Theorem 5.9 in [19], $C_{B S E}(\Delta(A))=\operatorname{Lip}_{1}(\mathbb{R})$. 
Now, it is not difficult to construct functions $f$ in $\operatorname{Lip}_{1}(\mathbb{R}) \cap C_{0}(\mathbb{R})$ such that $\rho_{R}(f)$ does not converge to zero as $R \rightarrow \infty$. For instance, let $f(t)=0$ for $t<0$ and $f(t)=\frac{t}{4}$ for $0 \leq t \leq 2$, and for all $n \geq 2$, define $f$ on the interval $[n, n+1]$ by

$$
f(t)=\left\{\begin{array}{ccc}
\frac{1}{n}+(t-n) & \text { for } & n \leq t \leq n+\frac{1}{n} \\
\frac{2}{n}+\left(t-\frac{n^{2}+1}{n}\right) \frac{n}{(n+1)^{2}} & \text { for } & n+\frac{1}{n} \leq t \leq n+1
\end{array}\right.
$$

Using that the ascent of $f$ on each interval $\left[n, n+\frac{1}{n}\right], n \geq 2$, is one, it is straightforward to check that $\rho_{R}(f)=1$ for all $R \geq 2$. Thus

$$
f \in \operatorname{Lip}_{1}(\mathbb{R}) \cap C_{0}(\mathbb{R}) \text { and } f \notin A \text {. }
$$

It follows from Theorem 4.8 that $A_{e}$, which is isomorphic to the subalgebra of $\operatorname{Lip}_{1}(\mathbb{R}) \cap C_{0}(\mathbb{R})$ consisting of all $f$ for which the $\operatorname{limit}_{\lim } \rightarrow \infty \rho_{R}(f)$ exists, fails to be a BSE-algebra.

\section{ACKNOWLEDGEMENT}

The authors are grateful to the referee for several valuable comments and suggestions to improve the exposition.

\section{REFERENCES}

[1] L. Baggett and K. Taylor, A sufficient condition for the complete reducibility of the regular repesentation, J. Funct. Anal. 34 (1979), 250-265. MR552704 (81f:22005)

[2] F.F. Bonsall and J. Duncan, Complete normed algebras, Springer, New York, 1973. MR:0423029 (54:11013)

[3] J. Bourgain and H. Rosenthal, Applications of the theory of semi-embeddings to Banach space theory, J. Funct. Anal. 52 (1983), 149-188. MR707202 (85g:46018)

[4] C. Chou and G. Xu, The weak closure of the set of left translation operators, Proc. Amer. Math. Soc. 127 (1999), 465-470. MR1468187 (99c:43003)

[5] M. Cowling, The Fourier-Stieltjes algebra of a semisimple group, Colloq. Math. 41 (1979), 89-94. MR550633 (81e:43005)

[6] H.G. Dales, Banach algebras and automatic continuity, Oxford University Press, Oxford, 2000. MR1816726 (2002e:46001)

[7] R. Doss, On the transform of a singular or an absolutely continuous measure, Proc. Amer. Math. Soc. 19 (1968), 361-363. MR0222569 (36:5619)

[8] P. Eymard, L'algèbre de Fourier d'un groupe localement compact, Bull. Soc. Math. France 92 (1964), 181-236. MR0228628 (37:4208)

[9] A. Figà-Talamanca, Positive definite functions which vanish at infinity, Pacific J. Math. 69 (1977), 355-363. MR0493175 (58:12206)

[10] V. Flory, On the Fourier algebra of a locally compact amenable group, Proc. Amer. Math. Soc. 29 (1971), 603-606. MR0283138 (44:371)

[11] B.E. Forrest, Amenability and ideals in $A(G)$, J. Austral. Math. Soc. Ser. A 53 (1992), 143-155. MR1175708 (93i:43002)

[12] B.E. Forrest and M. Skantharajah, A note on a type of approximate identity in the Fourier algebra, Proc. Amer. Math. Soc. 120 (1994), 651-652. MR1166356 (94d:43001)

[13] B.E. Forrest, E. Kaniuth, A.T. Lau and N. Spronk, Ideals with bounded approximate identities in Fourier algebras, J. Funct. Anal. 203 (2003), 286-304. MR.1996874 (2004e:43002)

[14] C.C. Graham and O.C. McGehee, Essays in commutative harmonic analysis, Springer, New York, 1979. MR.550606 (81d:43001)

[15] F.P. Greenleaf, Invariant means on topological groups, van Nostrand, New York, 1969. MR0251549(40:4776)

[16] S. Grosser and M. Moskowitz, On central topological groups, Trans. Amer. Math. Soc. 127 (1967), 317-340. MR0209394 (35:292)

[17] B. Host, Le théorème des idempotents dans $B(G)$, Bull. Soc. Math. France 114 (1986), 215-223. MR860817 (88b:43003) 
[18] J. Inoue and S.-E. Takahasi, Constructions of bounded weak approximate identities for Segal algebras on LCA groups, Acta Sci. Math. (Szeged) 66 (2000), 257-271. MR1768865 (2001i:43004)

[19] J. Inoue and S.-E. Takahasi, On characterizations of the image of the Gelfand transform of commutative Banach algebras, Math. Nachr. 280 (2007), 105-126. MR 2290386 (2007m:46071)

[20] K. Izuchi, The Bochner-Schoenberg-Eberlein theorem and spaces of analytic functions on the open unit disc, Math. Japon. 37 (1992), 65-77. MR1148517 (93b:46044)

[21] C.A. Jones and C.D. Lahr, Weak and norm approximate identities are different, Pacific J. Math. 72 (1977), 99-104. MR0447972 (56:6282)

[22] E. Kaniuth, A.T. Lau and A. Ülger, Homomorphisms of commutative Banach algebras and extensions to multiplier algebras with applications to Fourier algebras, Studia Math. 183 (2007), 35-62. MR2360256 (2008k:46147)

[23] I. Khalil, Sur l'analyse harmonique du groupe affine de la droite, Studia Math. 51 (1974), 139-167. MR0350330 (50:2823)

[24] R. Larsen, An introduction to the theory of multipliers, Springer-Verlag, New York, 1971. MR0435738 (55:8695)

[25] J.R. Liukkonen and M.W. Mislove, Symmetry in Fourier-Stieltjes algebras, Math. Ann. 217 (1975), 97-112. MR0420148(54:8163)

[26] V. Losert, Properties of the Fourier algebra that are equivalent to amenability, Proc. Amer. Math. Soc. 92 (1984), 347-354. MR759651 (86b:43010)

[27] G. Mauceri and M.A. Picardello, Non-compact unimodular groups with purely atomic Plancherel measure, Proc. Amer. Math. Soc. 78 (1980), 77-84. MR.548088 (81h:22005)

[28] C. Nebbia, Multipliers and asymptotic behaviour of the Fourier algebra of nonamenable groups, Proc. Amer. Math. Soc. 84 (1982), 549-554. MR643747(83h:43002)

[29] T.W. Palmer, Banach algebras and the general theory of $*$-algebras, Vol. I, Cambridge University Press, Cambridge, UK, 1994. MR1270014 (95c:46002)

[30] J.-P. Pier, Amenable locally compact groups, Wiley Interscience, New York, 1984. MR767264 (86a:43001)

[31] W. Rudin, Fourier analysis on groups, Wiley Interscience, New York, 1962. MR0152834 $(27: 2808)$

[32] V. Runde and N. Spronk, Operator amenability of Fourier-Stieltjes algebras. II, Bull. London Math. Soc. 39 (2007), 194-202. MR.2323448(2008i:46043)

[33] E.L. Stout, The theory of uniform algebras, Bogden and Quigley, New York, 1971. MR0423083 (54:11066)

[34] S.-E. Takahasi and O. Hatori, Commutative Banach algebras which satisfy a BochnerSchoenberg-Eberlein-type theorem, Proc. Amer. Math. Soc. 110 (1990), 149-158. MR.1017008 (90m:46086)

[35] S.-E. Takahasi and O. Hatori, Commutative Banach algebras and BSE-inequalities, Math. Japonica 37 (1992), 47-52. MR.1176031 (93h:46069)

[36] S.-E. Takahasi, Y. Takahashi, O. Hatori and K. Tanahashi, Commutative Banach algebras and BSE-norm, Math. Japonica 46 (1997), 273-277. MR.1479824 (98f:46044)

[37] A. Ülger, Multipliers with closed range on commutative Banach algebras, Studia Math. 153 (2002), 59-80. MR 1948928 (2003k:43004)

[38] A. Ülger, Some results about the spectrum of commutative Banach algebras under the weak topology and applications, Monatsh. Math. 121 (1996), 353-379. MR1389676 (98a:46058)

Institut für Mathematik, Universität Paderborn, D-33095 Paderborn, Germany

E-mail address: kaniuth@math.uni-paderborn.de

Department of Mathematics, Koc University, 34450 Sariyer, Istanbul, Turkey

E-mail address: aulger@ku.edu.tr 